\title{
Supplementary Information for \\ Engineering bacteria to catabolize the carbonaceous component of sarin: teaching $E$. coli to eat isopropanol
}

Margaret E. Brown, Aindrila Mukhopadhyay, and Jay D. Keasling

\section{Supplementary Results}

\section{Abbreviations}

Figure S1. Lysate reactivity of E. coli expressing ACX complex

Figure S2. Promoter and sequence modification for ACX protein complex expression S4

Table S1. Plasmids used in this study

Table S2. Plasmid depictions for ACX operon construction

Figure S3. SDS-PAGE analysis of alcohol dehydrogenase expression and solubility S7

Figure S4. Purification of recombinant ACX complex from E. coli DH1(DE3) S8

Figure S5. Kinetic assays surveying activity of the recombinant ACX S9

Figure S6. Plasmid system for growth of E. coli on IPA S10

Figure S7. HPLC analysis of linearity in IPA and acetone detection S10

Figure S8. Growth of plasmid-bearing E. coli BW25113(DE3) on IPA S11

Figure S9. Increasing IPTG induction concentrations leads to lower IPA-dependent growth S12

Figure S10. Expanding the IPA degradation system to include overexpression of the carbonic anhydrase S13

Figure S11. Supplementation with thiamine or extra metals show no growth benefit $\quad \mathrm{S} 14$

Figure S12. Solubility of ACX protein as induced with increasing concentrations of IPTG inducer

Figure S13. Purification of MMS-grown ACX $\$ S 16$

Table S3. Oligonucleotides used for plasmid construction $\quad \mathrm{S17}$

Table S4. G-blocks used for gene assembly $\quad$ S19

Literature cited $\quad$ S25 


\section{Abbreviations}

$\mathrm{ADH}$, alcohol dehydrogenase

ACX, acetone carboxylase

AACS, acetoacetyl-CoA synthetase

AACT, acetoacetyl-CoA thiolase

PTE, phosphotriesterase

PDE, phosphodiesterase

$\mathrm{PHN}$, phosphonate degradation cluster

$\mathrm{CA}$, carbonic anhydrase

XAacx, acetone carboxylase from Xanthobacter autotrophicus PY2

AAacx, acetone carboxylase from Aromatoleum aromaticum PY2

PFadh (PF), alcohol dehydrogenase from Pseudomonas fluorescens

CBadh (CB), alcohol dehydrogenase from Clostridium beijerinckii

GSadh2 (GS), alcohol dehydrogenase from Gordonia sp. str. TY-5

TBadh (TB), alcohol dehydrogenase from Thermoanaerobacter brockii

RNaacs (RN), acetoacetyl-CoA synthetase from Rattus norvegicus

ECaact (EC), acetoacetyl-CoA thiolase from E. coli

IPA, isopropanol

IPTG, isopropyl $\beta$-D-1-thiogalactopyranoside

HPLC, high-performance liquid chromatography

TPP, thiamine pyrophosphate

TMP, thiamine monophosphate

$\mathrm{T}$, thiamine

MM, minimal media

MMS, minimal media with supplements

LB, lysogeny broth

RFP, red fluorescent protein

Glc, glucose

aTet, anhydrotetracycline 
Figure S1. Lysate reactivity of $E$. coli expressing ACX complex. HPLC traces of ACX reactivity with acetone shows the production of acetoacetate only in the presence of the ACX and acetone. +ACX, ACX-expressing lysate; -ACX, control lysate.

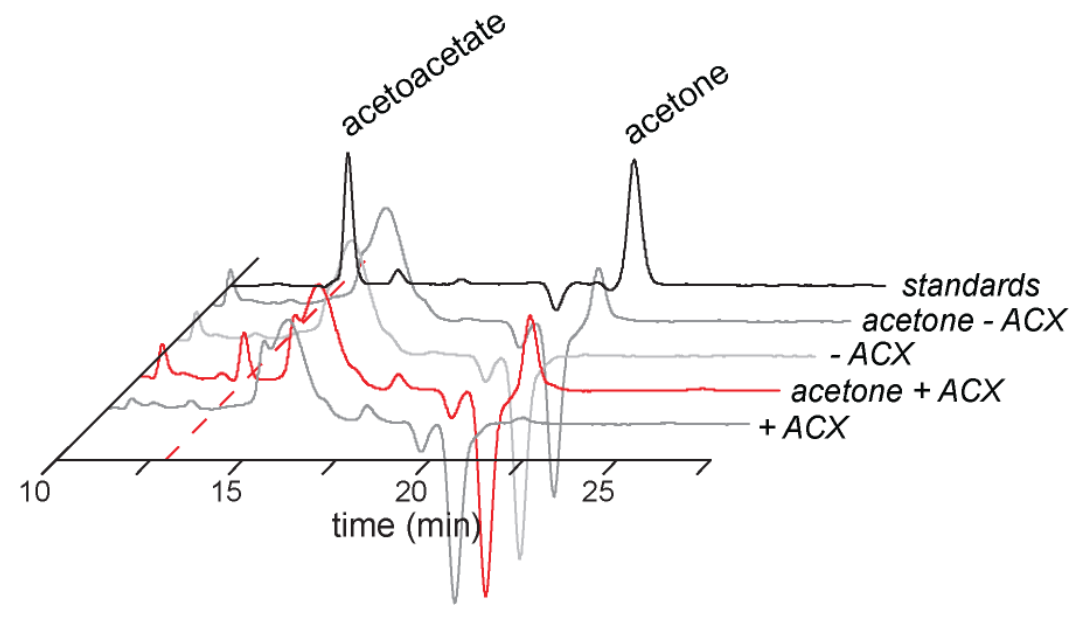


Figure S2. Promoter and sequence modification for ACX protein complex expression. A) Codon-modified acx sequences transcribed from different promoters, comparing the original expression off of the Trc-encoded pTrc33 and the BglBrick plasmid promoters (Trc, lacUV5, T7, ara). ${ }^{1}$ Modification of promoter shows little alteration in expression levels. B) Use of the native acx sequence in expression plasmids shows an increase in protein content when expressed from the T7 promoter of the pBbE7c vector. Lane 1, ACX; 2, ACXn; 3, ACXnT; 4, ACXnT-rbs; 5, ACXnT-dT7; 6, ACXnT-dT7-ssC; 7, ACXnT-dT7-v3; 8, RFP). Final modification (ACXnT-dT7v3) placing the native $C$ subunit in front of the native $A$ subunit for expression yields expression levels of all three subunits similar to that found in acetone-grown Xanthobacter. Blue arrows indicate the 3 subunits. C) Densitometric analysis of the 3 subunits of ACXnT-dT7-v3 as determined by ImageJ. Density, intensity measurement of band; size, molecular weight of subunit (Da).

A

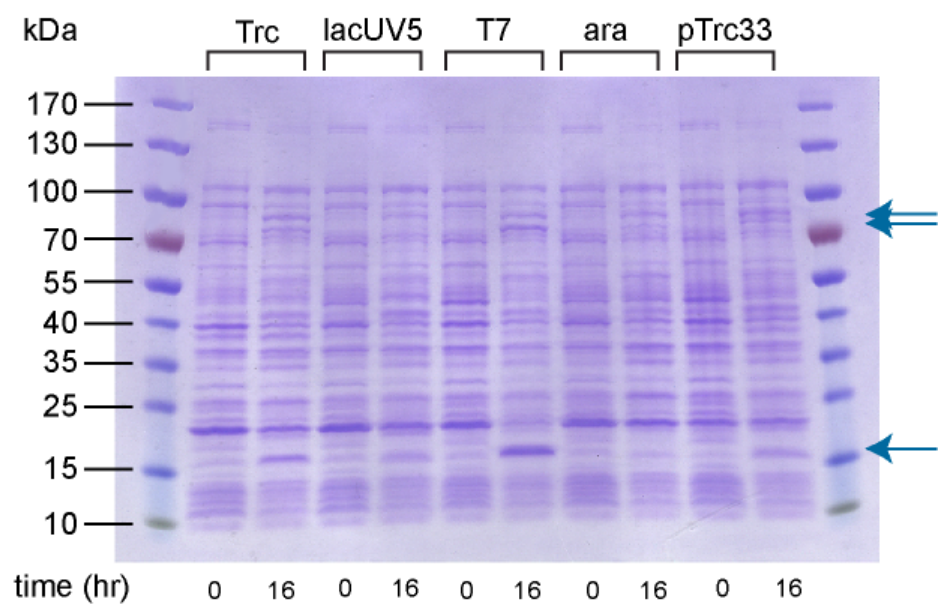

B

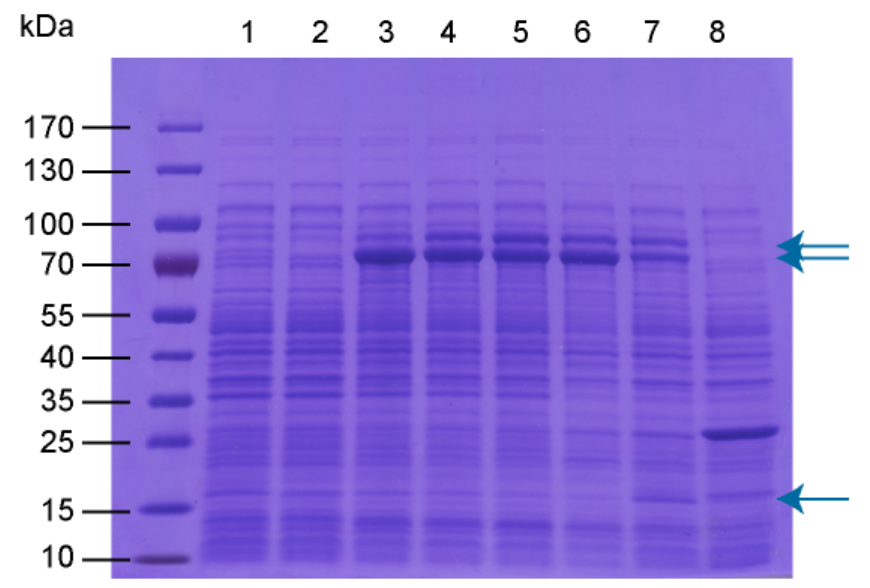

C

\begin{tabular}{lccc}
\hline subunit & density (D) & size (S) & ratio (D/S) \\
\hline acxA & 1372.9 & 78509 & 0.0175 \\
acxB & 1714.4 & 86342 & 0.0199 \\
acxC & 298.4 & 19774 & 0.0151 \\
\hline
\end{tabular}


Table S1. Plasmids used in this study.

\begin{tabular}{|c|c|c|c|c|}
\hline Strain & E. coli host & Plasmid 1 & Plasmid 2 & Strain number \\
\hline $1^{a}$ & $\mathrm{DH} 1(\mathrm{DE} 3)$ & pTrc33-empty** & - & - \\
\hline $2^{a}$ & DH1(DE3) & pTrc33-XAacx & - & JBEI-14106 \\
\hline $3^{a}$ & DH1(DE3) & pTrc33-AAacx & - & JBEI-14107 \\
\hline $4^{a}$ & DH1(DE3) & pET16b-RFP & - & JBEI-14108 \\
\hline $5^{a}$ & DH1(DE3) & pET16b-PFadh & - & JBEI-14102 \\
\hline $6^{a}$ & DH1(DE3) & pET16b-CBadh & - & JBEI-14103 \\
\hline $7^{a}$ & DH1(DE3) & pET16b-GSadh2 & - & JBEI-14104 \\
\hline $8^{a}$ & DH1(DE3) & pET16b-TBadh & - & JBEI-14105 \\
\hline $9^{a}$ & DH1(DE3) & pET16b-ECaact & - & JBEI-14101 \\
\hline $10^{a}$ & DH1(DE3) & pET16b-RNaacs & - & JBEI-14100 \\
\hline $11^{a}$ & DH1(DE3) & pBbE1c-XAacx & - & JBEI-14098 \\
\hline $12^{a}$ & DH1(DE3) & pBbE5c-XAacx & - & JBEI-14096 \\
\hline $13^{a}$ & DH1(DE3) & pBbE7c-XAacx & - & JBEI-14097 \\
\hline $14^{a}$ & DH1(DE3) & pBbE8c-XAacx & - & JBEI-14113 \\
\hline $15^{a}$ & DH1(DE3) & pBbE7c-XAacxn & - & JBEI-14112 \\
\hline $16^{a}$ & DH1(DE3) & pBbE7c-XAacxnT & - & JBEI-14118 \\
\hline $17^{a}$ & DH1(DE3) & pBbE7c-XAacxnT-rbs & - & JBEI-14206 \\
\hline $18^{a}$ & DH1(DE3) & pBbE7c-XAacxnT-dT7 & - & JBEI-14205 \\
\hline $19^{a}$ & DH1(DE3) & pBbE7c-XAacxnT-dT7-ssC & - & JBEI-14117 \\
\hline $20^{a}$ & DH1(DE3) & pBbE7c-XAacxnT-dT7-v3 & - & JBEI-14116 \\
\hline 21 & DH1(DE3) & pBbE7c-XAacxnT-dT7-v3 & pBbS5a-RFP & JBEI-14132 \\
\hline 22 & DH1(DE3) & pBbE7c-XAacxnT-dT7-v3 & pBbS5a-PF & JBEI-14133 \\
\hline 23 & DH1(DE3) & pBbE7c-XAacxnT-dT7-v3 & pBbS5a-CB & JBEI-14127 \\
\hline 24 & DH1(DE3) & pBbE7c-XAacxnT-dT7-v3 & pBbS5a-PF-CA & JBEI-14128 \\
\hline 25 & DH1(DE3) & pBbE7c-XAacxnT-dT7-v3 & pBbS5a-CB-CA & JBEI-14129 \\
\hline 26 & DH1(DE3) & pBbE7c-XAacxnT-dT7-v3 & pBbS5a-CB-CA-RN-EC & JBEI-14130 \\
\hline 27 & DH1(DE3) & pBbE7c-XAacxnT-dT7-v3 & pBbS2a-RFP & JBEI-14131 \\
\hline 28 & DH1(DE3) & pBbE7c-XAacxnT-dT7-v3 & pBbS2a-CB-CA & JBEI-14123 \\
\hline 29 & DH1(DE3) & pBbE7c-XAacxnT-dT7-v3 & pBbS2a-CB-CA-RN-EC & JBEI-14124 \\
\hline 30 & BW25113(DE3) & pBbE7c-XAacxnT-dT7-v3 & pBbS5a-RFP & JBEI-14125 \\
\hline 31 & BW25113(DE3) & pBbE7c-XAacxnT-dT7-v3 & pBbS5a-PF & JBEI-14126 \\
\hline 32 & BW25113(DE3) & pBbE7c-XAacxnT-dT7-v3 & pBbS5a-CB & JBEI-14121 \\
\hline
\end{tabular}

\begin{tabular}{lccc}
\hline Plasmid & Promoter & Origin of Replication & Antibiotic resistance \\
\hline pTrc33- $^{*}$ & Trc & p15a & $\mathrm{CmR}$ \\
pET16b-* & T7 & ColE1 & $\mathrm{CbR}$ \\
pBbE1c-* & Trc & ColE1 & $\mathrm{CmR}$ \\
pBbE5c- & lacUV5 & ColE1 & $\mathrm{CmR}$ \\
pBbE7c- & T7 & ColE1 & $\mathrm{CmR}$ \\
pBbE8c- & arabinose & ColE1 & $\mathrm{CmR}$ \\
pBbS5a- & lacUV5 & SC101 & $\mathrm{CbR}$ \\
pBbS2a- & Tet & SC101 & $\mathrm{CbR}$ \\
\hline
\end{tabular}

${ }^{*}=$ multiple constructs, ${ }^{* *}=$ no insert, ${ }^{a}=$ permanent storage in $\mathrm{DH} 10 \mathrm{~B}-\mathrm{T} 1^{\mathrm{R}}$ 
Table S2. Plasmid depictions for ACX operon construction.

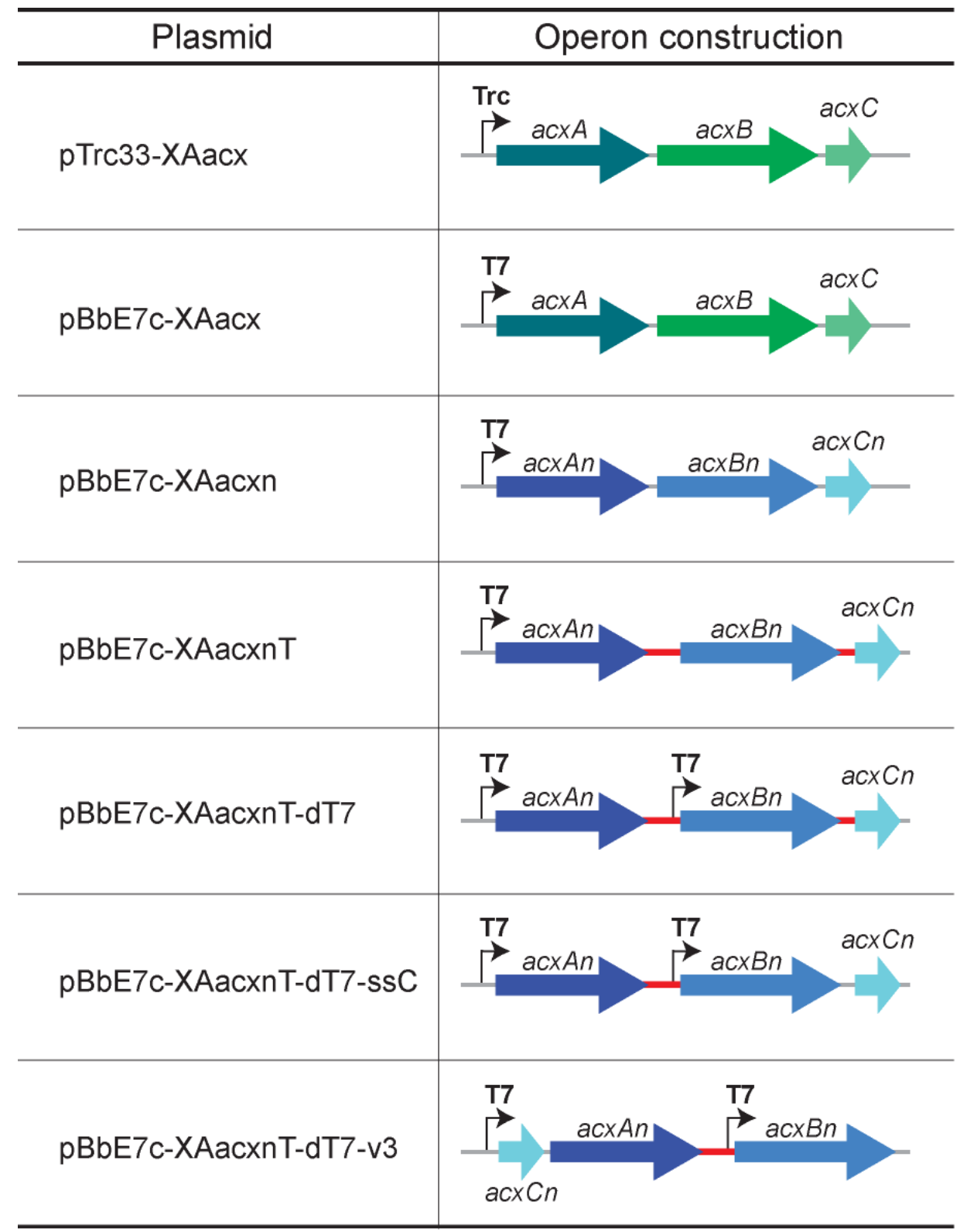

synthetic sequence

Xanthobacter native sequence 
Figure S3. SDS-PAGE analysis of alcohol dehydrogenase expression and solubility. Expression of the four candidate alcohol dehydrogenases in E. coli DH1(DE3) using $500 \mu \mathrm{M}$ IPTG. For ADHs: TB, Thermoanaerobacter brockii; CB, Clostridium beijerinckii; PF, Pseudomonas fluorescens; GS, Gordonia sp. str. TY-5. T, total lysate; S, soluble lysate.

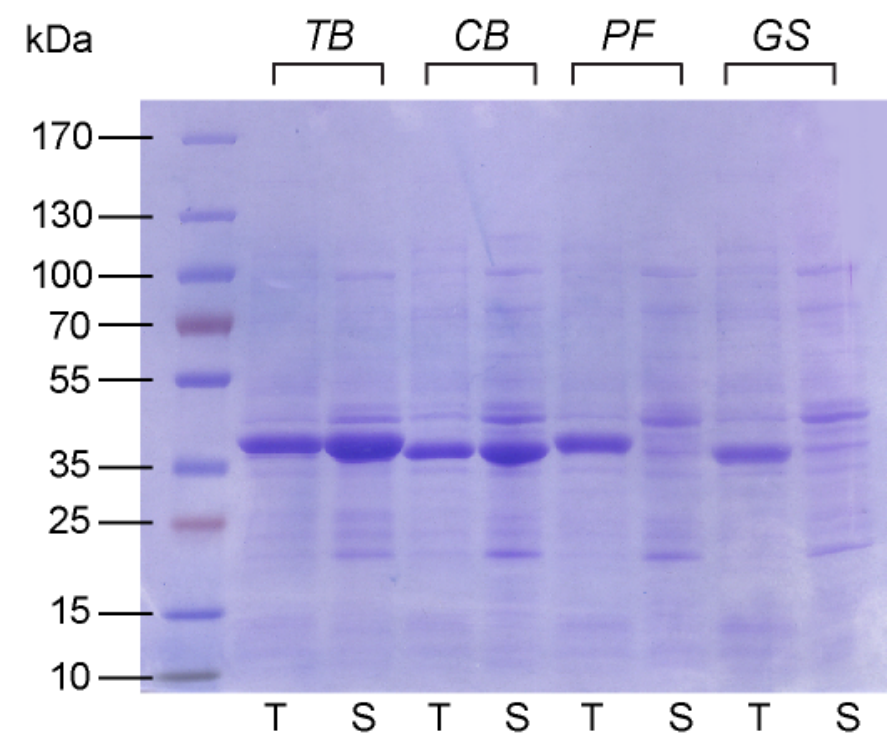


Figure S4. Purification of recombinant ACX complex from E. coli DH1(DE3). ACX was expressed in $1 \mathrm{~L}$ of both LB and MM- $0.8 \%$ glc using $500 \mu \mathrm{M} \mathrm{IPTG}$ as the inducing agent. A) FPLC traces for each purification using $5 \mathrm{~mL}$ DEAE resin. B) FPLC traces showing further separation of pooled fractions using size-exclusion chromatography (SEC). C) SDS-PAGE analyzing purity of pooled fractions from each purification stage. Red bars indicate pooled fractions of purification and analysis. T, total cell lysate; S, soluble lysate; P1, DEAE purification results; P2, SEC purification results. Blue arrows indicate the 3 subunits. D) Densitometric analysis of the purified ACX as determined by Image J. Density, intensity measurement of band; size, molecular weight of subunit (Da). MM, minimal medium.

A

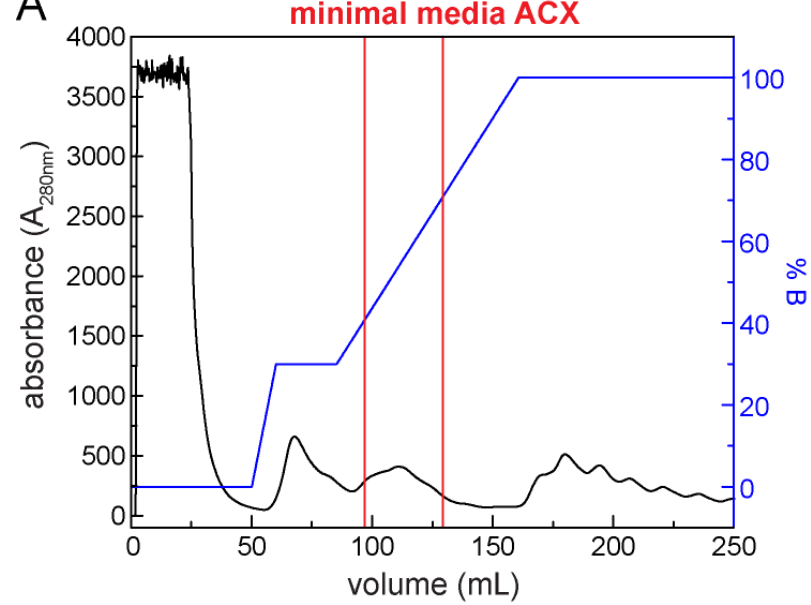

B

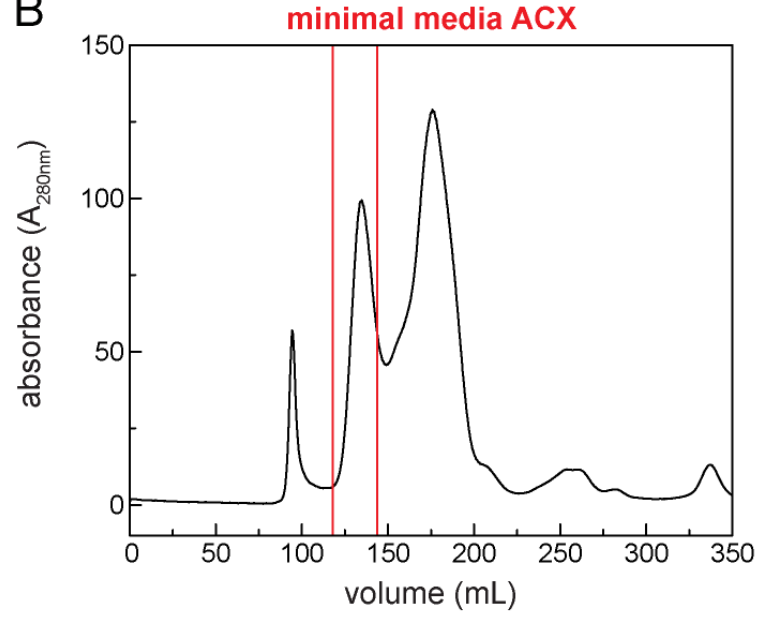

C

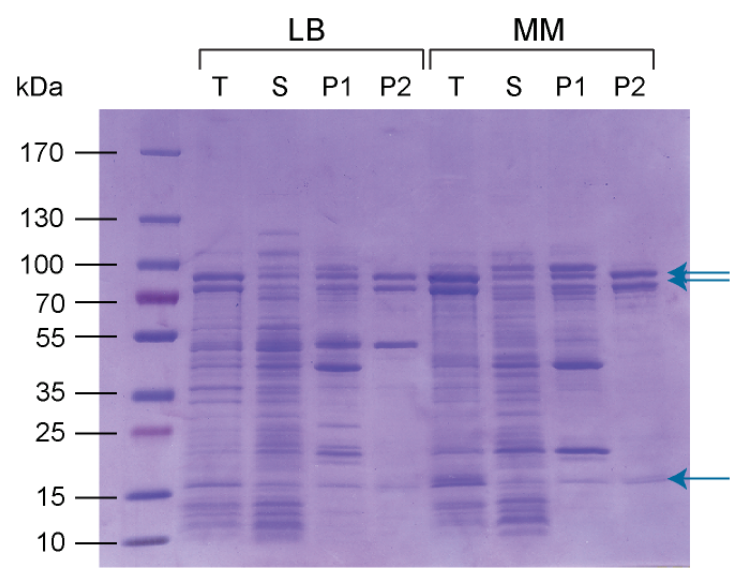

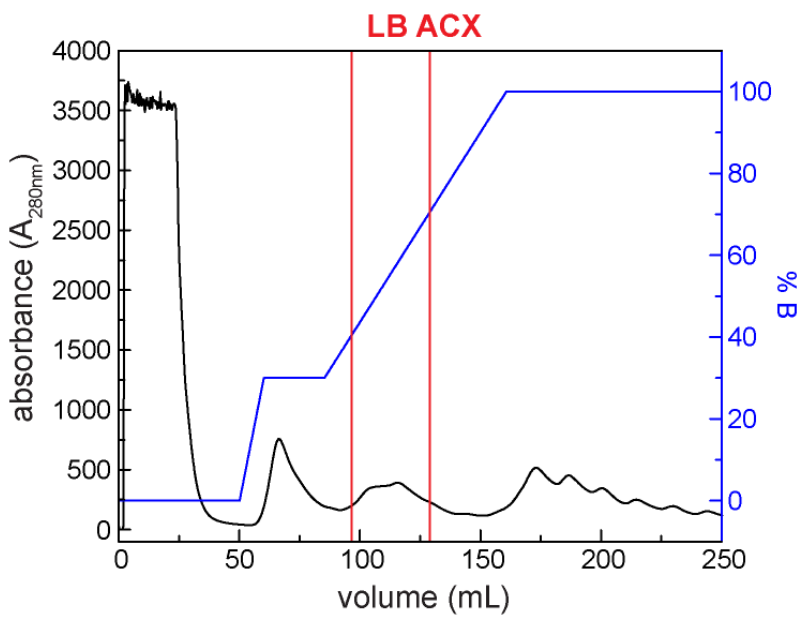

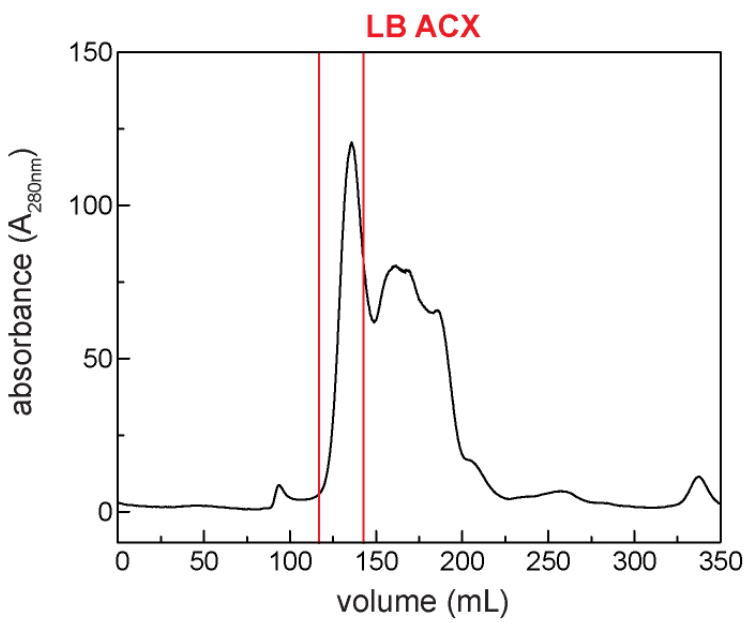

D

LB

\begin{tabular}{lccc}
\hline & density (D) & size (S) & ratio (D/S) \\
\hline acxA & 1179.6 & 78509 & 0.0150 \\
acxB & 1306.6 & 86342 & 0.0151 \\
acxC & 304.1 & 19774 & 0.0154 \\
\hline
\end{tabular}

MM

\begin{tabular}{lccc}
\hline & density (D) & size (S) & ratio (D/S) \\
\hline acxA & 1582.6 & 78509 & 0.0202 \\
acxB & 1559.7 & 86342 & 0.0181 \\
acxC & 421 & 19774 & 0.0213 \\
\hline
\end{tabular}


Figure S5. Kinetic assays surveying activity of the recombinant ACX. Enzymatic data is in triplicate. Reactions monitored the oxidation of $\mathrm{NADH}$ at $340 \mathrm{~nm}$. A) Using an acetoacetate standard, a coupled kinetic assay using the NADH-dependent 3-hydroxybutyrate dehydrogenase to monitor acetoacetate presence was verified. B) The activity of purified ACX (from growth in LB) against acetone to produce acetoacetate was monitored using this coupled kinetic assay at $340 \mathrm{~nm}$. C) Monitoring the effect of adding thiamine analogues $(1 \mathrm{mM})$ to the reactions of ACX against acetone. SA, specific activity; -, no additional supplement; $T$, thiamine; TMP, thiamine monophosphate; TPP, thiamine pyrophosphate.

A

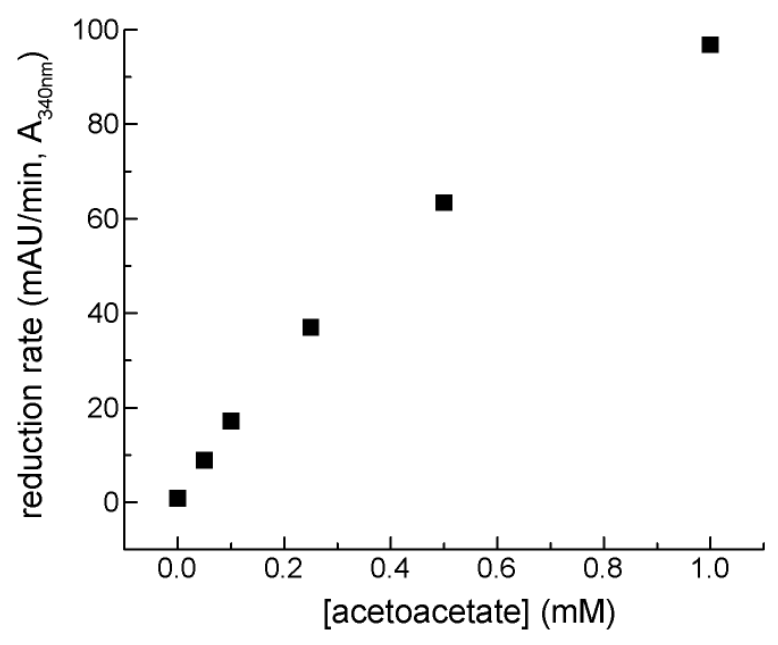

B

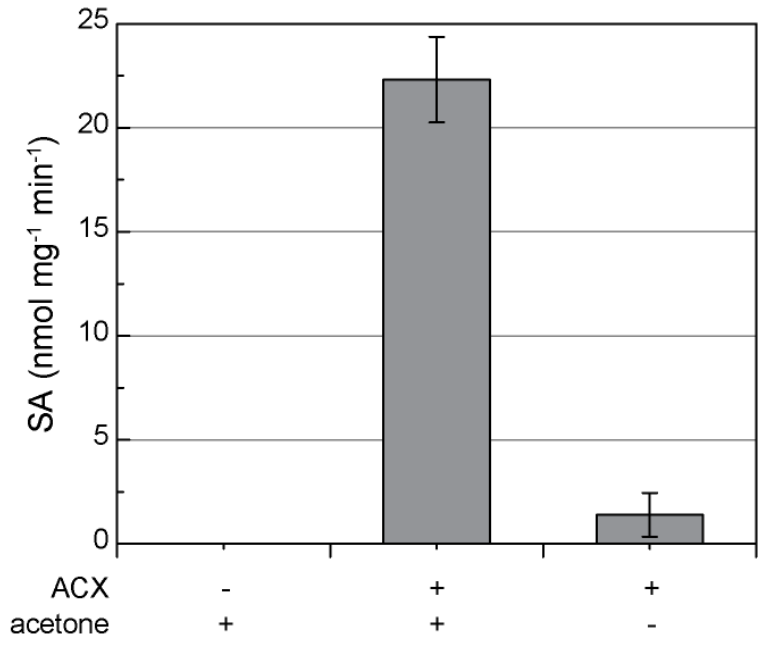

C

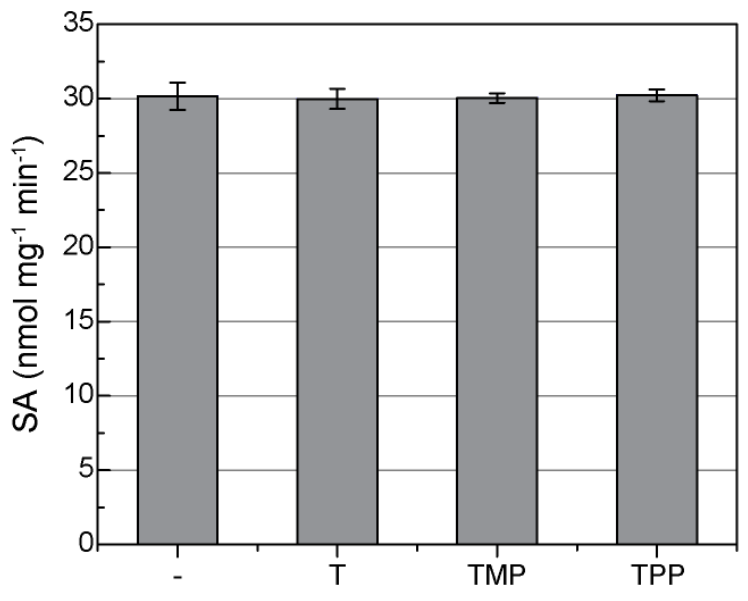


Figure S6. Plasmid system for growth of E. coli on IPA.
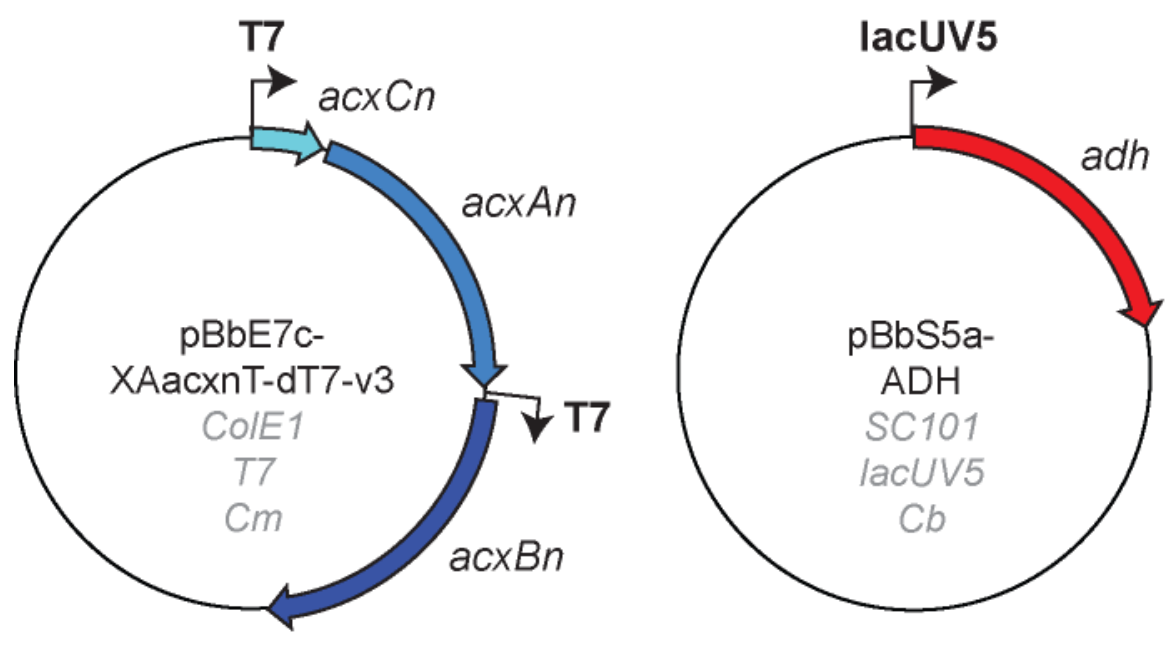

Figure S7. HPLC analysis of linearity in IPA and acetone detection. Concentrations used include $500 \mathrm{mM}, 100 \mathrm{mM}, 10 \mathrm{mM}$, and $2.5 \mathrm{mM}$. Black squares, IPA; red points, acetone; lines are best fit.

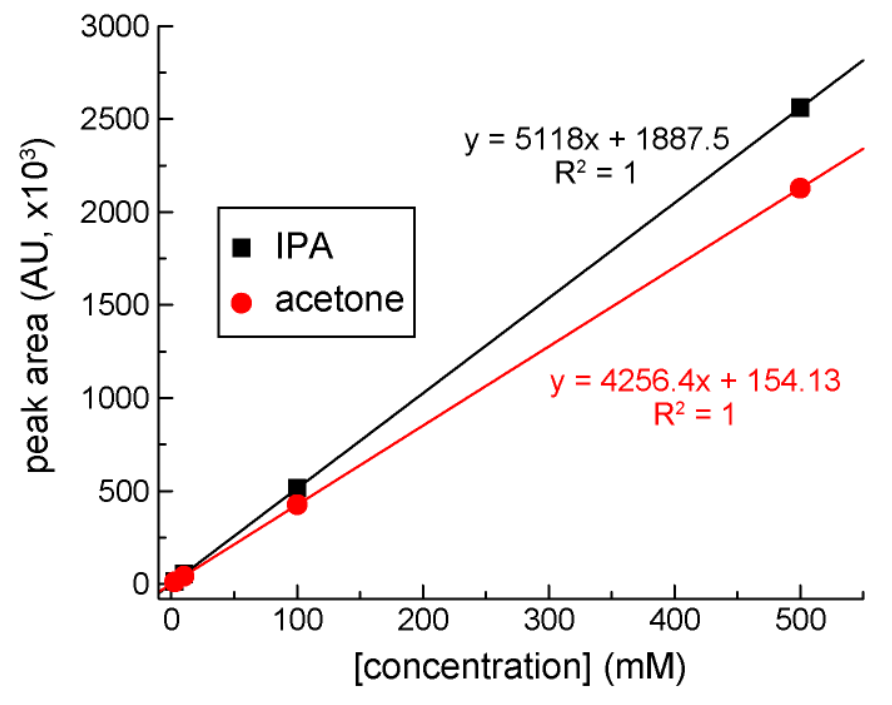


Figure S8. Growth of plasmid-bearing E. coli BW25113(DE3) on IPA. A) BW25113(DE3) carrying pBbE7c-XAacxnT-dT7-v3 and either an alcohol dehydrogenase (JBEI-14126, JBEI14121 ) or red fluorescent protein (JBEI-14125). Growth in supplemented minimal media in the presence or absence of isopropanol. B) HPLC analysis of day 6 isopropanol concentrations in extracellular medium. RFP, red fluorescent protein; PF, PFadh; CB, CBadh; IPA, 0.75\% isopropanol; glc, $0.2 \%$ glucose.

A

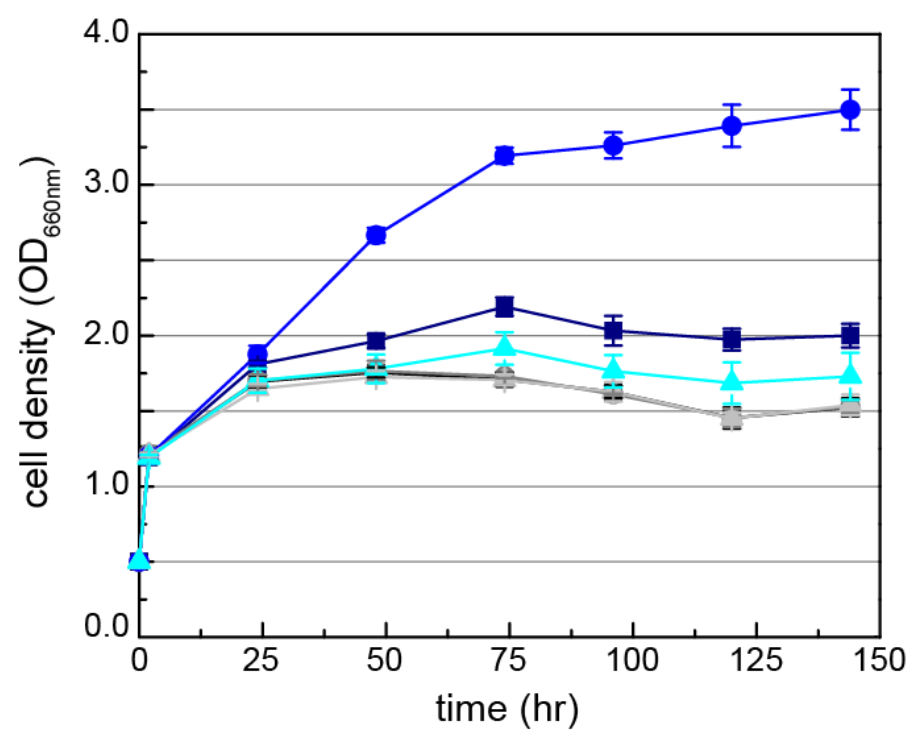

$\mathrm{B}$

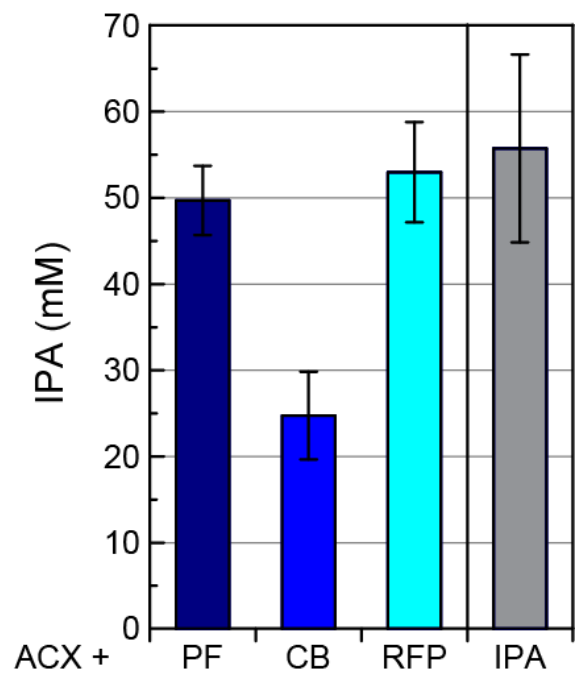

$0.2 \%$ glc $\left[\begin{array}{l}-A C X+\text { pBbS5a-PF } \\ -A C X+\text { pBbS5a-CB } \\ -A C X+\text { pBbS5a-RFP }\end{array}\right.$

$0.2 \%$ glc + IPA $\left[\begin{array}{l}\rightarrow-A C X+p B b S 5 a-P F \\ -A C X+p B b S 5 a-C B \\ -A C X+p B b S 5 a-R F P\end{array}\right.$ 
Figure S9. Increasing IPTG induction concentrations leads to lower IPA-dependent growth. A) Growth of E. coli DH1(DE3) carrying the pBbE7c-XAacxnT-dT7-v3 + CBadh plasmid system (JBEI-14127) with increasing inducer concentrations of IPTG. B) HPLC analysis of day 4 isopropanol concentrations in extracellular medium. C) HPLC analysis of day 4 acetone concentrations in extracellular medium. IPA, $0.75 \%$ isopropanol; glc, $0.2 \%$ glucose. D) Daily analysis of IPA consumption using no cell, IPA-only media as control. Transparent bars show IPA concentrations used to determine fraction of IPA remaining.
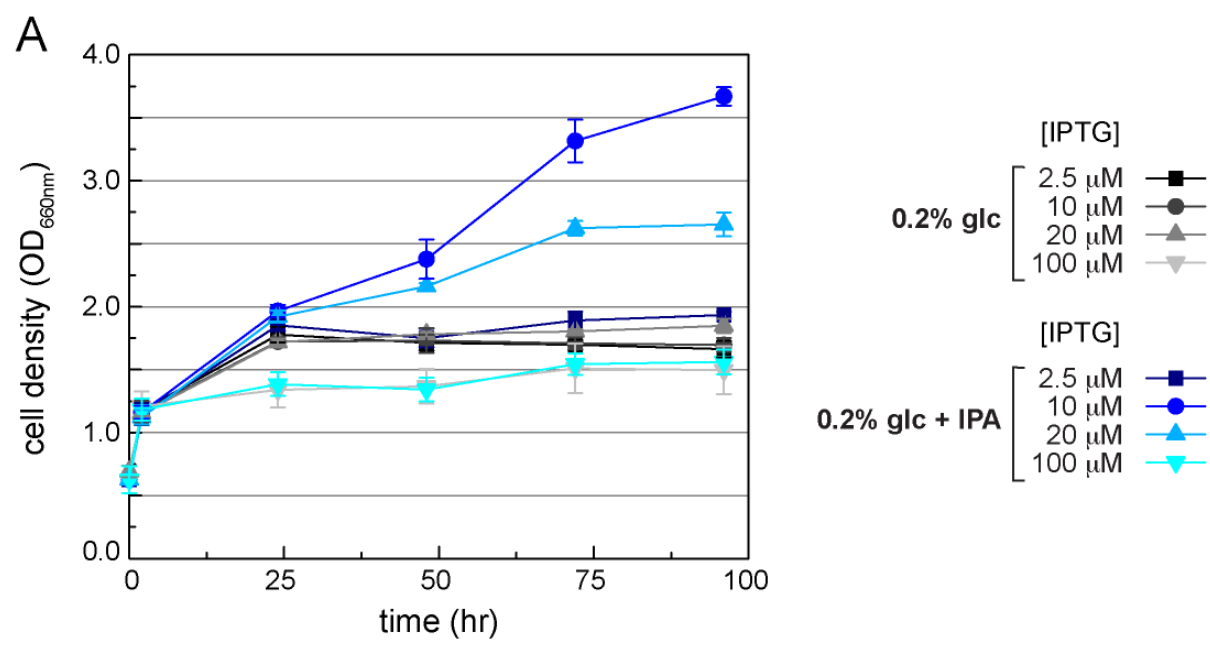

B

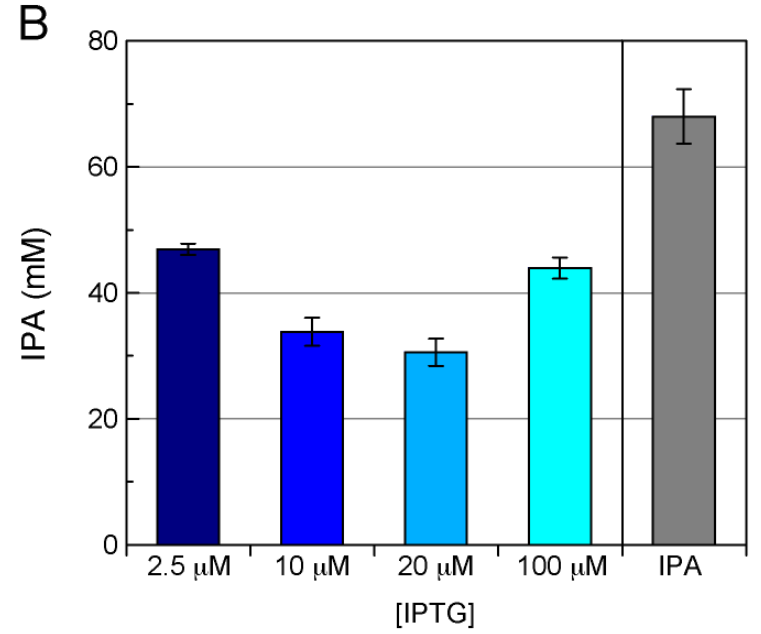

C

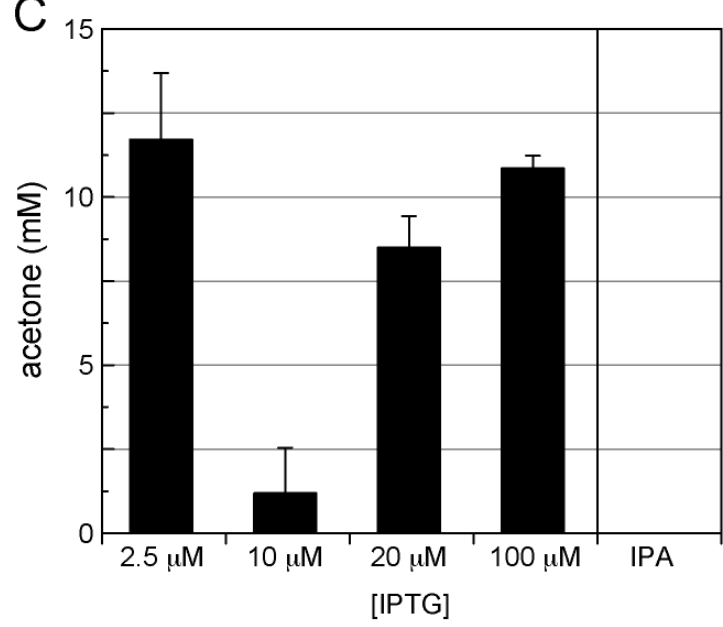

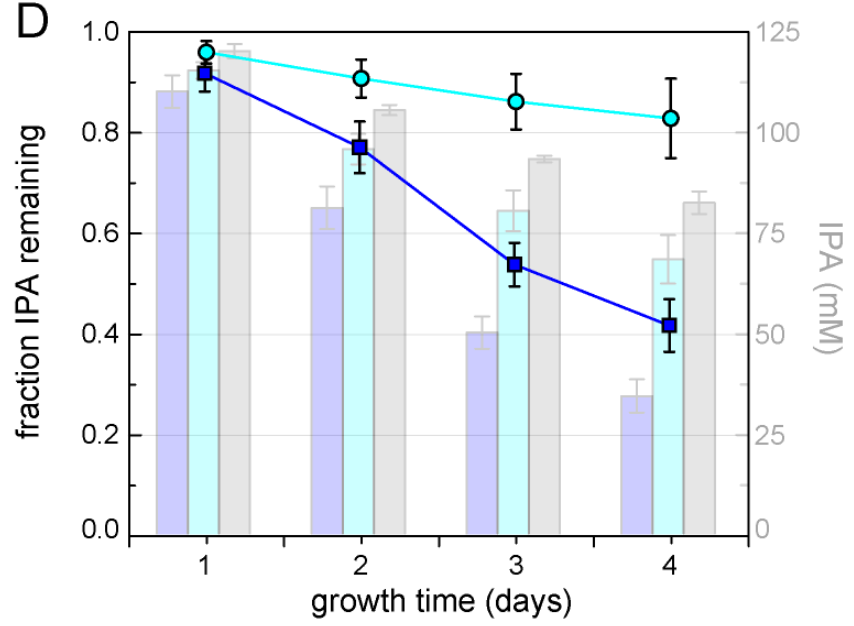


Figure S10. Expanding the IPA degradation system to include overexpression of the carbonic anhydrase. A) Growth curves of E. coli DH1(DE3) carrying the pBbE7c-XAacxnTdT7-v3 + alcohol dehydrogenase plasmids with (JBEI-14128, JBEI-14129) and without (JBEI14133, JBEI-14127) the carbonic anhydrase. B) HPLC analysis of day 4 isopropanol concentrations in extracellular medium. PF, PFadh; CB, CBadh; CA, carbonic anhydrase; IPA, $0.75 \%$ isopropanol; glc, $0.2 \%$ glucose.

A

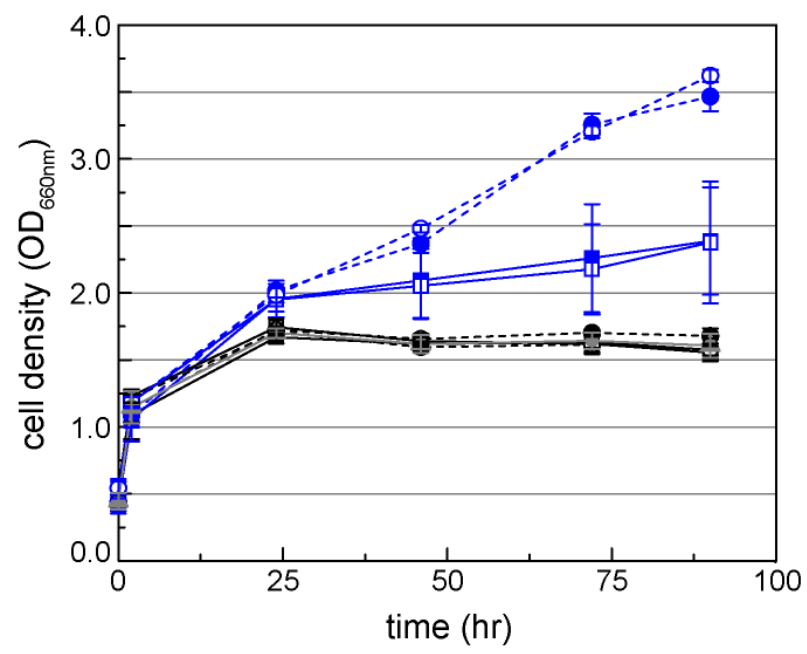

$\mathbf{0 . 2 \%}$ glc $\left[\begin{array}{l}--A C X+p B b S 5 a-P F \\ -\square-A C X+p B b S 5 a-P F-C A \\ -\bullet-A C X+p B b S 5 a-C B \\ -0-A C X+p B b S 5 a-C B-C A\end{array}\right.$
B

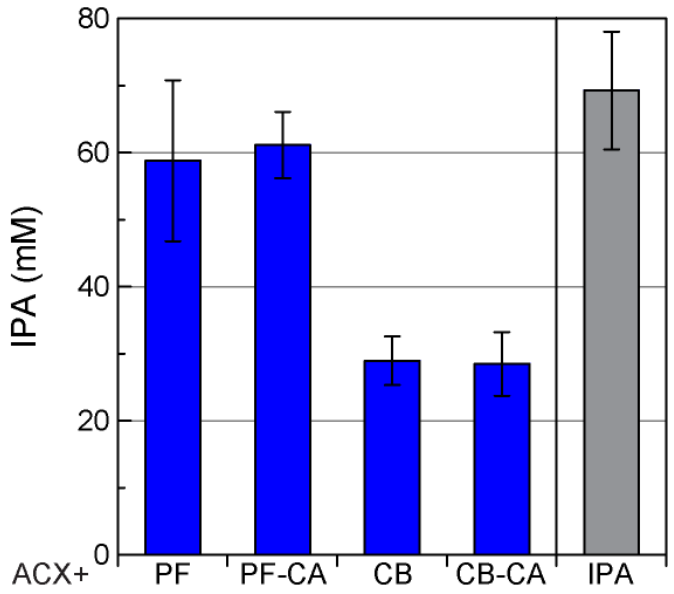

$\mathbf{0 . 2} \%$ glc + IPA $\left[\begin{array}{l}- \\ --A C X+p B b S 5 a-P F \\ --A C X+p B b S 5 a-P F-C A \\ --A-A C X+p B b S 5-C B \\ --C B-C A\end{array}\right.$ 
Figure S11. Supplementation with thiamine or extra metals show no growth benefit. A) Growth of E. coli DH1(DE3) carrying the pBbE7c-ACXnT-dT7-v3 + pBbS5a-CB-CA plasmids (JBEI-14129). Induction of system at either 10 or $20 \mu \mathrm{M}$ IPTG and supplementing with extra thiamine (T, $0.05 \mu \mathrm{g} / \mathrm{mL})$. B) Growth of E. coli DH1(DE3) carrying the pBbE7c-ACXnT-dT7-v3 + pBbS5a-CB-CA-RN-EC plasmids (JBEI-14130) induced with $20 \mu \mathrm{M}$ IPTG and supplementing with extra metals (concentrations below). CB, CBadh; CA, carbonic anhydrase; IPA, 0.75\% isopropanol; glc, 0.2\% glucose; MM, minimal medium; MMS, supplemented minimal medium; T, thiamine.

A
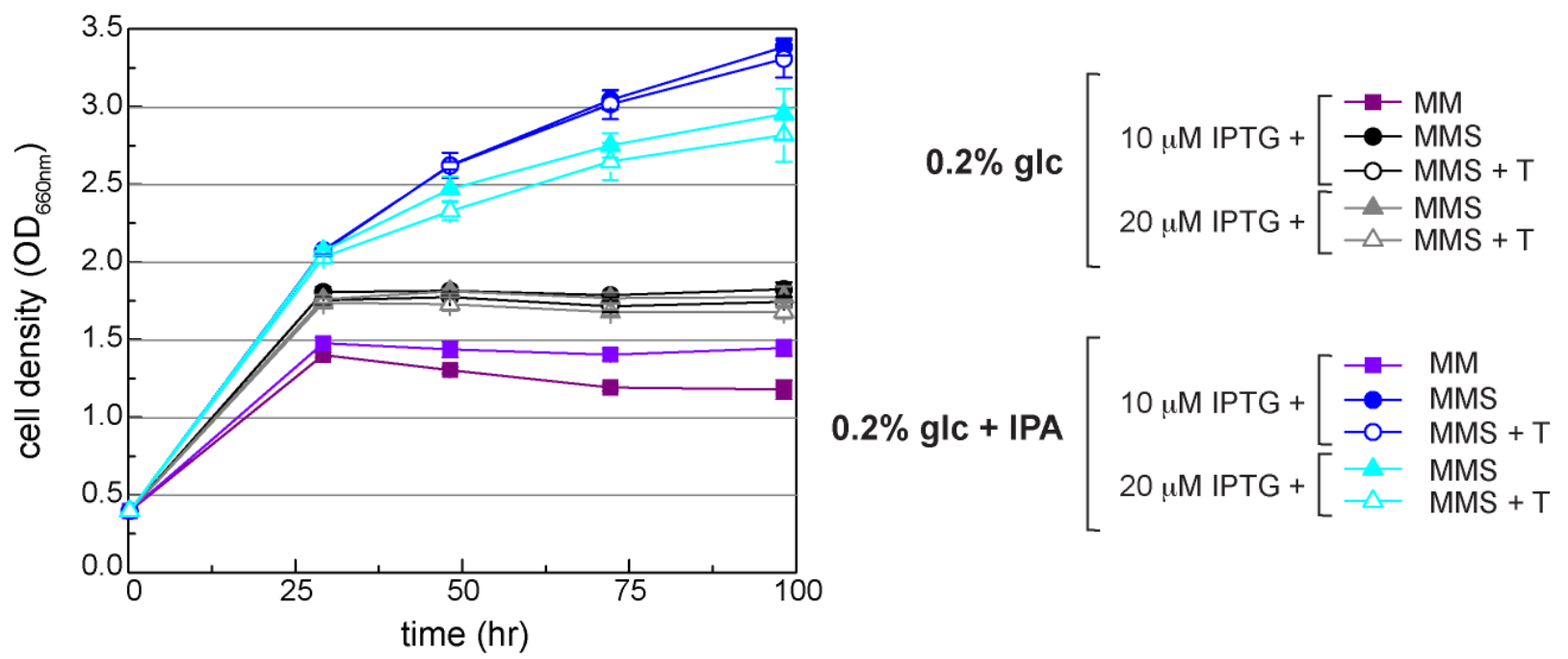

B
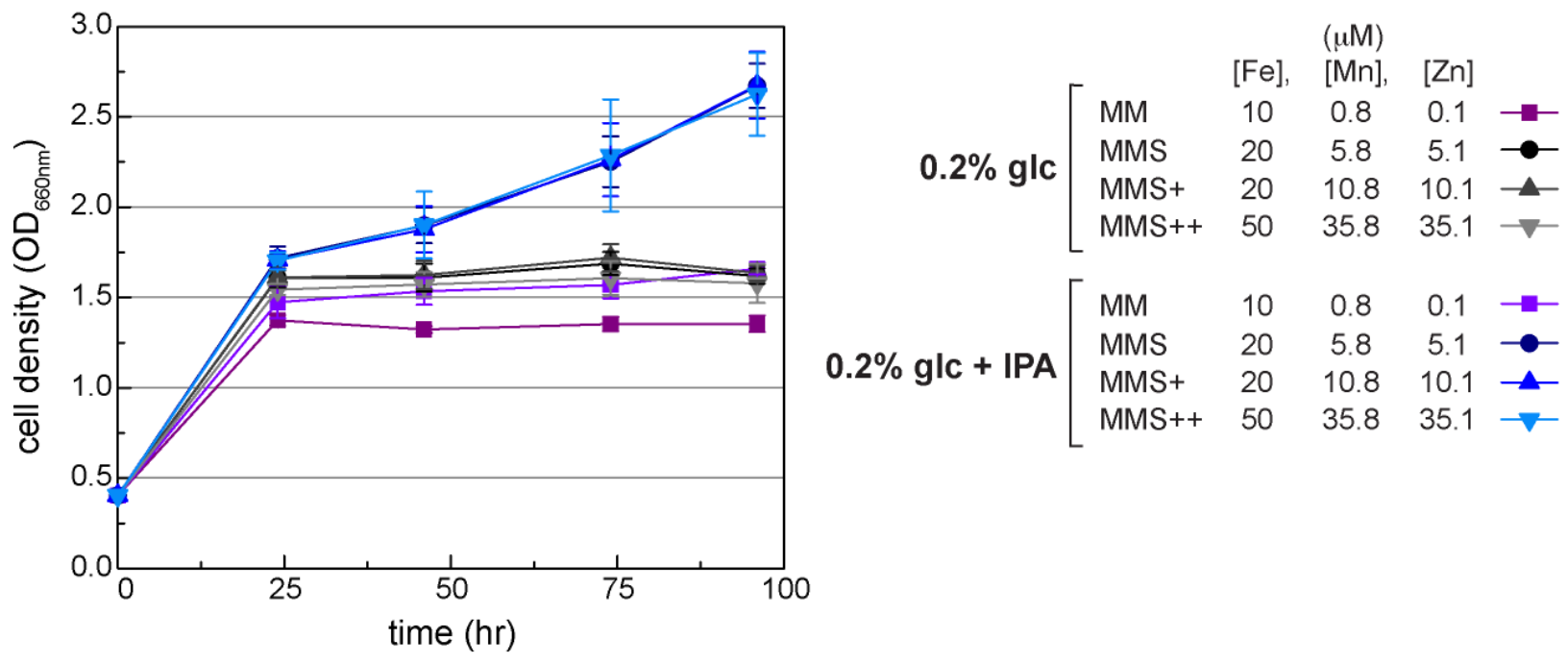
Figure S12. SDS-PAGE analysis of ACX protein solubility as induced with increasing concentrations of IPTG inducer. T, total lysate; S, soluble lysate. Blue arrows denote the 3 subunits of $A C X$.

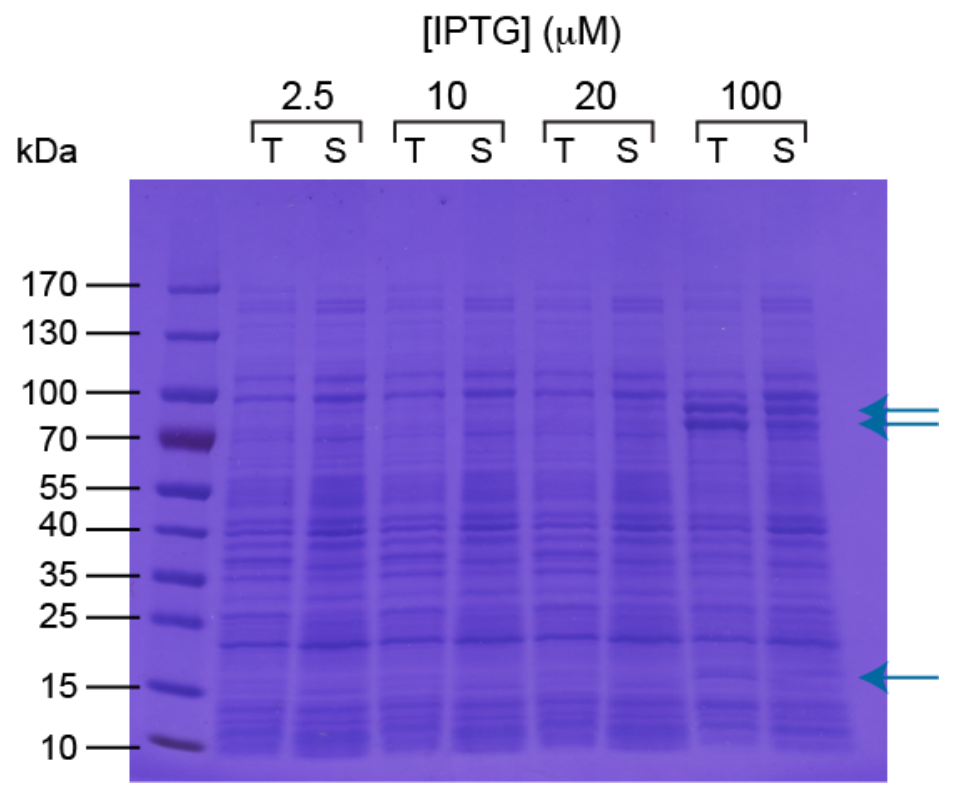


Figure S13. Purification of MMS-grown ACX. ACX purification from $1 \mathrm{~L}$ of MMS- $0.8 \%$ glC using $10 \mu \mathrm{M}$ IPTG induction. A) FPLC trace for the purification with DEAE resin. B) FPLC trace showing further separation using size-exclusion chromatography. C) SDS-PAGE analyzing purity of each purification stage. Red bars indicate pooled fractions for next purification step. T, total cell lysate. S, soluble lysate. P1, DEAE purification results. P2, SEC purification results. Blue arrows indicate the 3 subunits. D) Kinetic activity analysis and comparison of all purified ACX protein on acetone. SA, specific activity. E) Kinetic analysis of MMS ACX activity against acetone with supplemental thiamine analogs. -, no supplement; T, thiamine; TMP, thiamine monophosphate; TPP, thiamine pyrophosphate; SEC, size-exclusion chromatography.
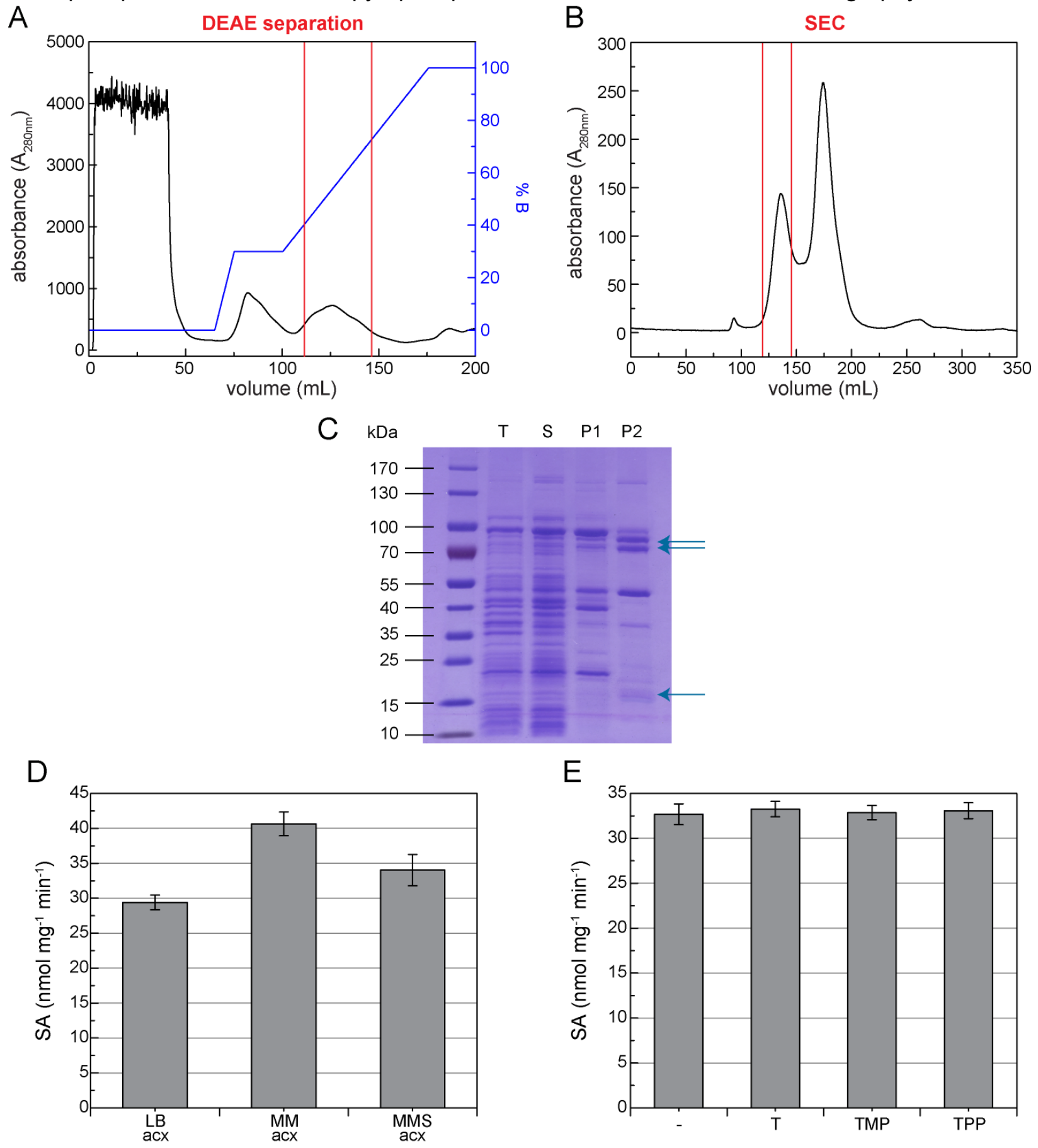
Table S3. Oligonucleotides used for plasmid construction.

\begin{tabular}{|c|c|}
\hline Name & Sequence \\
\hline TBadh-F1 & ctagaaataattttgtttaactttaagaaggagatataCCatgaagggattcgctatg \\
\hline TBadh-R1 & cagtagcgccataatactttgccgcatcaacacataccgg \\
\hline TBadh-F2 & ccggtatgtgttgatgcggcaaagtattatg \\
\hline TBadh-R2 & ttaagccaggatgaccactggtttaatcagg \\
\hline CBadh-F1 & ctagaaataatttgtttaactttaagaaggagatataCCatgaaaggctttgcgatg \\
\hline CBadh-R1 & cacgcggtccacacctttgccgttagtaagtttcattacttgg \\
\hline CBadh-F2 & agtaatgaaacttactaacggcaaaggtgtggac \\
\hline CBadh-R2 & USE AAaas-R3 \\
\hline PFadh-F1 & ctagaaataatttgtttaactttaagaaggagatataCCatggctacgatgaaagc \\
\hline PFadh-R1 & catcaacaccgataatggtgcttgcaccctttaaac \\
\hline PFadh-F2 & gcacgtttaaagggtgcaagcaccattatcggtgttgatgctgtcgc \\
\hline PFadh-R2 & USE AAaas-R3 \\
\hline AAaas-R3 & cctttcgggctttgttagcagccggatcctcgagCATATGtta \\
\hline GSadh2-F1 & ctagaaataattttgtttaactttaagaaggagatataCCatgaaggcgctggtgtttc \\
\hline GSadh2-R1 & ccagaatctcttcacgccaaccagatgatgtcgagacaac \\
\hline GSadh2-F2 & gttgtctcgacatcatctggttggcgtgaa \\
\hline GSadh2-R2 & ttatgccgacatgatcacctttaaggctttgg \\
\hline XAacxA-F1 & gataacaatttcacacgagctcggtaccaggagatataCCatgaacgttccggtc \\
\hline XAacxA-R1 & gagtctggcgagaaggttccgctgcataaccttccagaat \\
\hline XAacxA-F2 & attctggaaggttatgcagcggaaccttct \\
\hline XAacxA-R2 & accaccgtaactgaagcagacgaaggatg \\
\hline XAacxA-F3 & ctactctccagcatccttcgtctgcttcagttacggtggtgccggaccagtacata \\
\hline XAacxA-R3 & ttggtcaacggtcacattcatggtatatctcctTCTAGAttaaacctcacggagatgaaagaggcgatgcc \\
\hline XAacxB-F1 & gaaggagatataccatgaatgtgaccgttgaccaaagtaccttagcag \\
\hline XAacxB-R1 & accccgcgatgcgggtacgctcgtctaacatccaatagcg \\
\hline XAacxB-F2 & cgctattggatgttagacgagcgtaccc \\
\hline XAacxB-R2 & acattcaaagctattaactgcgtgaatctcgtcgtattggttgaagc \\
\hline XAacxB-F3 & ccaatacgacgagattcacgcagttaatagctttg \\
\hline XAacxB-R3 & ttcacctgcgaagcgttccaagacataaccaccgttgata \\
\hline XAacxB-F4 & tatcaacggtggttatgtcttggaacgett \\
\hline XAacxB-R4 & cgggtatatgccatggtatatctcctttactcttcaacaaattgcacg \\
\hline $\mathrm{XAacxC}-\mathrm{F} 1$ & aagagtaaaggagatataccatggcatatacccgttcgaa \\
\hline XAacxC-R1 & atccgccaaaacagccaagcttgcatgcctgcaggtcgactta \\
\hline AAacxA-F1 & gataacaatttcacacgagctcggtaccaggagatataCCatggaagccctgcaagc \\
\hline AAacxA-R1 & cgatcgctcaccttcttcagggtctggcgagaaggttctg \\
\hline AAacxA-F2 & cagaaccttctcgccagaccctgaagaag \\
\hline AAacxA-R2 & gaagcccaccccttcggtatagccgtaagtatgcaccggt \\
\hline AAacxA-F3 & accggtgcatacttacggctataccgaag \\
\hline AAacxA-R3 & tcagcatattcatggtatatctccttctagattatttcacttctttcaggtgaaacaggc \\
\hline AAacxB-F1 & agtgaaataaTCTAGAaggagatataccatgaatatgctgaccaataaagaagttg \\
\hline AAacxB-R1 & tgccttcccaaaaaatcggcacgatggtagcgatgtcaca \\
\hline AAacxB-F2 & tgtgacatcgctaccatcgtgccgatttttt \\
\hline AAacxB-R2 & tggtgagtgcatgatgctgtccagtttggcgaatgcattg \\
\hline AAacxB-F3 & caatgcattcgccaaactggacagcatca \\
\hline AAacxB-R3 & caatcactattcatataaccattgcccatgaaaaacatggtcca \\
\hline AAacxB-F4 & ccatgtttttcatgggcaatggttatatgaatagtgattggggtatg \\
\hline AAacxB-R4 & cgtatacgttgacatggtatatctcctttattcttctacctggaccaccgtg \\
\hline
\end{tabular}




\begin{tabular}{|c|c|}
\hline AAacxC-F1 & ggtagaagaataaaggagatataccatgtcaacgtatacgaacgaacaggtggc \\
\hline AAacxC-R1 & atccgccaaaacagccaagcttgcatgcctgca \\
\hline XAacx_F & /5phos/atgaacgttccggtcgga \\
\hline XAacx_R & /5phos/ttacgcatccgcacgttc \\
\hline pBbEXC_F & /5phos/ggatccaaactcgagtaagga \\
\hline pBbEXC_R & /5phos/atgtatatctccttcttaaaagatcttttga \\
\hline E1C_A-Bridging & caatttcagaattcaaaagatcttttaagaaggagatatacatatgaacgttccggtcggacac \\
\hline E1C_B-Bridging & ggaacgtgcggatgcgtaaggatccaaactcgagtaaggatctcca \\
\hline $\mathrm{E} 5 \mathrm{C}$ & use $\mathrm{E} 1 \mathrm{C} A \& B$ \\
\hline E7C & use E1C A\&B \\
\hline E8C_A-Bridging & gggaattcaaaagatcttttaagaaggagatatacatatgaacgttccggtcggacac \\
\hline E8C_B & use E1C_B \\
\hline XAacxAn-F6 & aattcaaaagatcttttaagaaggagatatacatatgaacgttcccgtgggacacctgc \\
\hline XAacxAn-R4 & agtcacgttcatggtatatctccttctagatcacacctcgcgcaggtggaacag \\
\hline XAacxBn-F5 & gtgtgatctagaaggagatataccatgaacgtgactgttgaccagagcaccc \\
\hline XAacxBn-R5 & tcgagcgggtataggccatggtatatctccttcattcctccacgaactgcacggtgtg \\
\hline $\mathrm{XAacxCn-F2}$ & gaggaatgaaggagatataccatggcctatacccgctcgaagatcgtcg \\
\hline $\mathrm{XAacxCn-R4}$ & ttgatgcctggagatccttactcgagtttggatcctcaggcgtcggcccgctcgg \\
\hline XAacxAn-R5 & aacagtcacgttcatattgattcctcctttgagttcgttgactcggattgagcgtgc \\
\hline XAacxBn-F6 & aacgaactcaaaggaggaatcaatatgaacgtgactgttgaccagagcaccc \\
\hline XAacxBn-R6 & taggccatggtatatctcctattgattttgttttccaaggaaaaaatgggcggcagcg \\
\hline XAacxCn-F3 & tggaaaacaaaaatcaataggagatataccatggcctatacccgctcgaagatcgtcg \\
\hline XAacxAn-R6 & cgctcacaattcccctatagtgagtcgtattattgagttcgttgactcggattgagcgtg \\
\hline XAacxAn-R7 & tactaagatggggaattgttatccgctcacaattcccctatagtgagtcgtattattgag \\
\hline XAacxAn-R8 & ctcctcttatacttaactaatatactaagatggggaattgttatccgctcacaattcccc \\
\hline XAacxBn-R6 & taggccatggtatatctcctattgattttgttttccaaggaaaaaatgggcggcagcg \\
\hline XAacxBn-F7 & tatattagttaagtataagaggaggaatcaatatgaacgtgactgttgaccagagcaccc \\
\hline XAacxAn-F8 & tgaagatcttataagaaggaggtattcatatgaacgttcccgtgggacacctgc \\
\hline XAacxAn-R9 & gggtgctctggtcaacagtcacgttcat \\
\hline XAacxBn-F8 & atgaacgtgactgttgaccagagcaccc \\
\hline XAacxBn-R7 & gcctggagatccttactcgagtttggatcctcattcctccacgaactgcacggtgtg \\
\hline XAacxCn-F4 & caaaagatcttttaagaaggagatatacatatggcctatacccgctcgaagatcgtcg \\
\hline XAacxCn-R5 & catatgaatacctccttcttataagatcttcaggcgtcggcccgctcgg \\
\hline PFadh-F5 & taacaatttcatttaagaaggagatatacatatggctacgatgaaagcagccat \\
\hline PFadh-F6 & acaatttcagaattcaaaagatctttaagaaggagatatacatatggctacg \\
\hline PFadh-R5 & tgcctggagatccttactcgagtttggatccttacggagtaatcgccactttcatcaca \\
\hline CBadh-F3 & gaattcaaaagatcttttaagaaggagatatacatatgaaaggctttgcgatgctgg \\
\hline CBadh-R3 & ctggagatccttactcgagtttggatccttataaaatcaccactgctttaatcaggtctt \\
\hline CA-F1 & aaattaagaaggagatataccatgaaagacatagatacactcatca \\
\hline CA-R4 & agatccttactcgagtttggatccttatttgtggttggcgtgtttc \\
\hline CA-F4 & aagtggcgattactccgtaaggatccaaattaagaaggagatataccatg \\
\hline CA-F5 & aagcagtggtgattttataaggatccaaattaagaaggagatataccatg \\
\hline pTet-F1 & cagtgatagagaaaagaattcaaaagatcttttaagaaggagatatacat \\
\hline ECaat-F3 & caagacttttaaagatctaggagataatccatgaaaaattgtgtcatcgtcagtgcggta \\
\hline ECaat-R8 & cctggagatccttactcgagttaattcaaccgttcaatcaccatc \\
\hline RNaas-R6 & gattatctcctagatctttaaaagtcttgtaattccggaatatcgcgatagagatccagg \\
\hline RNaas-F7 & aataaggatccaaactcgagaattattataagaaggaggtataccatgtccaaactcgca \\
\hline
\end{tabular}


Table S4. G-blocks used for gene assembly.

\begin{tabular}{|c|c|}
\hline Name & Sequence \\
\hline $\begin{array}{l}\text { TBadh } \\
\text {-G1 }\end{array}$ & 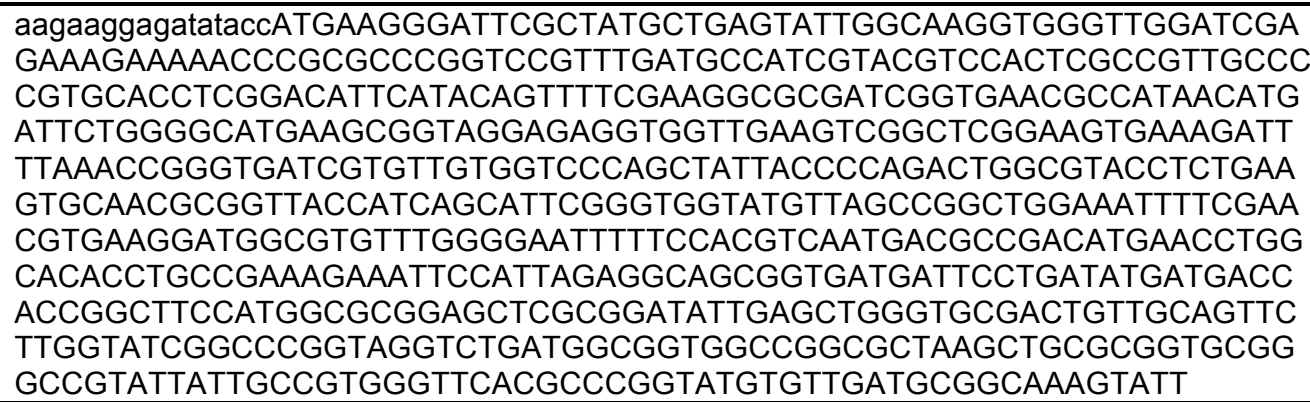 \\
\hline $\begin{array}{l}\text { TBadh } \\
\text {-G2 }\end{array}$ & $\begin{array}{l}\text { CCGGTATGTGTTGATGCGGCAAAGTATTATGGCGCTACTGATATCGTTAACTACAAAGAC } \\
\text { GGTCCGATTGAAAGCCAGATTATGAATCTCACGGAGGGCAAAGGAGTCGACGCAGCCAT } \\
\text { TATCGCTGGCGGTAATGCTGATATTATGGCAACAGCGGTCAAGATTGTGAAACCTGGCG } \\
\text { GAACCATTGCCAATGTGAACTATTTTGGCGAGGGCGAAGTGTTGCCAGTCCCCCGCCTG } \\
\text { GAATGGGGCTGCGGCATGGCACACAAGACCATTAAAGGTGGTCTGTGCCCTGGCGGCC } \\
\text { GTCTGCGTATGGAGCGTTTAATGACCTGGTATTTATAAACGCGTCGATCCGTCCAAAC } \\
\text { TGGTCACCCACGTCTTTCGTGGCTTTGATAACATCGAAAAGCATTTATGCTGATGAAAG } \\
\text { ATAAACCGAAAGACCTGATTAAACCAGTGGTCATCCTGGCTcatatgctcgaggatccggctgctaac } \\
\text { aaa }\end{array}$ \\
\hline
\end{tabular}

CBadh aagaaggagatataccATGAAAGGCTTTGCGATGCTGGGTATTAATAAGCTGGGCTGGATCGA

-G1 AAAAGAACGTCCCGTTGCTGGTTCGTATGATGCGATTGTTCGCCCCCTGGCGGTCAGCC CGTGTACCAGCGATATCCATACCGTCTTTGAAGGTGCCCTGGGCGATCGTAAAAACATG ATTTTAGGCCATGAAGCGGTGGGCGAAGTCGTGGAAGTTGGGAGCGAAGTTAAAGACTT CAAACCAGGCGATCGCGTGATTGTCCCATGCACGACCCCCGACTGGCGTAGTCTGGAG GTGCAAGCGGGGTTTCAACAACACAGTAACGGCATGCTGGCCGGTTGGAAATTTAGCAA CTTTAAAGACGGCGTGTTCGGTGAATACTTTCATGTGAACGATGCTGACATGAACCTGGC AATCTTGCCGAAGGACATGCCTTTGGAAAATGCAGTAATGATTACGGATATGATGACGAC GGGTTTTCATGGCGCTGAACTGGCGGATATTCAGATGGGCTCAAGCGTTGTTGTTATCG GAATTGGTGCGGTAGGTCTGATGGGTATTGCTGGTGCCAAATTGCGCGGCGCCGGCCG TATCATTGGTGTAGGATCTCGTCCGATTTGCGTCGAGGCTGCAAAATTCTACGGGGCAA CTGATATTCTCAACTATAAAAACGGGCACATTGTCGACCAAGTAATGAAACTTACTAACG GCAAAGGTGTG

CBadh AGTAATGAAACTTACTAACGGCAAAGGTGTGGACCGCGTGATCATGGCCGGGGGCGGA -G2 AGTGAGACTTTGTCGCAAGCTGTATCCATGGTTAAGCCGGGCGGTATCATTAGTAATATT AACTACCACGGGAGCGGCGATGCCCTGTTAATTCCGCGTGTGGAGTGGGGTTGTGGCA TGGCGCATAAGACTATTAAGGGTGGTCTGTGTCCGGGTGGCCGCTTGCGCGCCGAAAT GCTGCGTGATATGGTGGTATATAACCGTGTTGACTTAAGTAAGTTAGTAACCCATGTGTA TCACGGTTTTGATCATATCGAGGAAGCACTTCTGCTGATGAAAGACAAACCAAAAGACCT GATTAAAGCAGTGGTGATTTTATAAcatatgctcgaggatccggctgctaacaaa

\begin{tabular}{ll} 
PFadh & aagaaggagatataccATGGCTACGATGAAAGCAGCCATTTTCGTCGAAAAAAATCGTATCGTG \\
-G1 & CTGGATGACAAACCGATCCGGAAGTTGTCCGTTAGACGCTTTGATTCGCATTACCACT \\
& ACGACCATTTGCGGACCGACGTTCATATTCTGCGCGGTGAGTATCCGGTTGCGAAAGG \\
& TCTTACAGTGGGTCACGAGCCAGTGGGTATTATCGAACGTCTGGGTAGCCAAGTTCGCG \\
& GGTTTGTTGAAGGACAGCGCGTGATTGCAGGCGCTATCACCCCGTCTGGTCAATCTTAT \\
& GCCTGCTTATGCGGCTGTGGAAGTCAGGATGGACCAGACACACGTCATGGCTTTCGCG \\
& CGATCGGTGGCTGGAAGTTCGGCAACACGATCGATGGTTGTCAGGCAGATATGTTCTT \\
& GTTCCGGATGCCCTGGCAAATCTGTGCCCAATTCCAGATGGCCTGAGCGATGAACAAGT \\
& GTTAATGTGTCCAGATATCATGTCTACTGGTTTTCAGGTGCAGAACGTGGTGAATGAG \\
& TATTGGTGATAGCGTGGCTGTGTTGCTTAGGTCCATTGGATTGGGGCAGTTGCGG \\
& GTGCACGTTTAAAGGGTGAAGCACCATTATCGGTGTTGATGCTGTCGC \\
\hline
\end{tabular}




\begin{abstract}
PFadh AGCACCATTATCGGTGTTGATGCTGTCGCGGAACGTATGACCGTGGCCCGCCGTCTGG -G2 GTGCGACACATGTCGTGAACTTTCGTGAGGGTGATGTGGTCGAACAAATCATGGCCTTG ACCGATGGTCGTGGCGTCGATGTGGCGATTGAAGCGCTGGGCACGCAGGGCACTTTCG AAAGTGCACTGCGTGTGCTGCGTCCGGGAGGCCGTTTATCTTCACTGGGTGTCTATTCA TCGGATCTCCGTATCCCGTTAGATGCGTTCGCAGCAGGTTTAGGTGACTACAGCATTGT GACCACCCTTTGCCCGGGTGGAAAAGAGCGTATGCGTCGCCTGATGGCAGTCGTACAG AGTGGAATGGTGGACCTGTCTCCCCTGGTTACTCATCGCTTCAAATTGGACGATATCGAA GCAGCGTACGATCTGTTCGCACACCAACGCGACGGTGTGATGAAAGTGGCGATTACTCC GTAAcatatgctcgaggatccggctgctaacaaa
\end{abstract}

\begin{tabular}{|c|c|}
\hline Sa & $\begin{array}{l}\text { aagaaggagatataccATGAAGGCGCTGGTGTTTCATGGGCCTGGTCAGAAAGCCTGGGAAGA } \\
\text { TGTTCCGGACCCGAAGTGTCCGAACCGACTGACGTAATTGTCAAAATGGATACGACCA } \\
\text { CTATTTGTGGTACGGACCTGCACATTCTGAAAGGCGATGTTCCAGCGGTTACCCCAGGT } \\
\text { CGCATCCTGGGTCATGAAGGTGTCGGTACTATCACGGAAGTGGGAGATGAGTACTAC } \\
\text { CCTGGCAGTTGGCACCAGGTGATCCTGTCTTGTATTCAAGTTGCGGAAATGCTCCTT } \\
\text { CTGCAAGCAGGGTGTACTCGCATTGCTCGGAGCGAGGACGTCAGGTATTGGTT } \\
\text { GGATCTTCGGTCACTTAATCGACGGTACGCAAGCGGAGACGTACGCGTCCGTACGCT } \\
\text { GAAAACAGTGTACATAAACTGCCGAGTGCCGTCTCACCGGAGCAGGGCACCCTGCTGA } \\
\text { GCGATATCCTGCCAACAGGTCATGAAATTGTGTGCGTCACGGTGCAGTGAAACCTGGT } \\
\text { GATGTGGTTGCGGTGATTGGCACTGGTCCCGTTGGCTTAGCTGCCATCGCAACGGCTG } \\
\text { GACTGTATGGACCGTCTCGCGTCATTGCTGTCGACATTGACGCCAATCGCGTGGAACAA } \\
\text { GCACGTCGCTTTGGTGCTACTGACGGGGTTGTCTCGACATCATCTGGTTGGCGTGAA }\end{array}$ \\
\hline 32 & $\begin{array}{l}\text { GTTGTCTCGACATCATCTGGTTGGCGTGAAGAGATTCTGGCGATGACCGATGGCTTAGG } \\
\text { CGTGGACGTCGCCATTGAAGCAGTGGGCATCCCAGACACTTTCCAAATGTGCCTGGACA } \\
\text { TTGTTCGTCCAGCAGGACACGTGGCTAATGTCGGTGTGCACGGCAAACCTGTCGAATTA } \\
\text { CCGATCCAGGATCTGTGGATTAGTAATATCGTGATGTCTATGGGCCTGGTGAACACCAAT } \\
\text { ACACTGGGTACACTGCTGAAACTTGTGGCACAGCGTCGCATTGATCCGGAGCCATTTAT } \\
\text { TAGCCATCGCTTTGGACTGGGTGAATTATCGATGCCTATGACGTCTTTAGCCGCGCTG } \\
\text { CCGAGACCAAAGCCTTAAAGGTGATCATGTCGGCAcatatgctcgaggatccggctgctaacaaa }\end{array}$ \\
\hline
\end{tabular}

AAaas gaaggagatataccATGAACTCGTACATGCCACAGGGATATGAATGGCGTACTACCCGTCTTG -G1 CACAGTTTATCGCCCGTCACGGCTATCGCGATCTGGATGATCTGCGTGAACGTGCAGCA CGCGAACCTGAACGCTTCTGGGACCAGGTCTTAGAGGCTATTGGCCTTGACTGGGCAAC TCCTTACCGTCAGACCCTGGACTTGACTGATGGTGTTATGTGGCCAAAATGGTTCGTTG GTGGACGTCTGGATTTAGTGGATAACTTGGTTGGTAAACATGCACGTGCCACCCCTGCG AAAATTGCGGTGCGTTGGGAGGGTGATGGAGGCGAACTGCGTACCTTAAGTTACGCAG CGCTCGCTGGTGAGGTCGAACGTGTTGCAGCAGGCCTTCGTGCGCTGGGAGTATCTGA AGGCGAGCGCATTGCGTTATATCTGCCGATGGTTGCCGAAGCAGTGGTGACAATGTTGG CAGCCACCCGTATCGGTGCGGTATTCATTCCATTTTTTTCAGGCTATGGTGCAGATTCTG TTGCGCAGCGTGTTGCGGATTGCGAAGCACGTGTGCTGGTTTGCGCGAATGGTTATTTC CGTCGTGGTAAACGCGTCCCCATGCTGGCAGACGCACGTGCAGCGGCTCGTGCCTGCC CATCTTTACGTCATCTGGTGGTGGTCGATC

AAaas CATCTTTACGTCATCTGGTGGTGGTCGATCGTCTTGGTCTGGGTCAGCTGGCAGCCAAC -G2 GATGGTGCCGCAGAGAGCTTTGCCGAGGTGGATTTTCGTGCCTTGCAGGCAGCGTCTC CTGATCGTGGACCAGCAAGCGCTTGGCCAGCAGATCAAACTTTAATGCTTATTTATACCA GCGGTACCACCGGTAAACCGAAAGGGGTGGTGCACACCCACGCAGGATTTCCGGTGAA GGCAGCACAAGACTTACTCATGGCATTCGACCTGCGTGCGGACGACACGCTCATGTGG GTTACCGATATGGGTTGGCTGATGGGTCCATGGATGGTGTATGGTGGTCTTATGCTGGG TGCCACACTGGTGCTGTATGAAGGGACCCCGGATTATCCGGATGCAGGTCGTTTGTGGC AAGTGGTTGAACGCCACGGTGTGACCCACTTTGGTCTGTCTCCGACACTGGTGCGTCTC CTGATGGCGAACGACGCCTCACTGCCAGCTCCGGGTGCGCTGGATACTTTACGCGTATT TGGCAGCACAGGCGAAGCATGGAACGAAGCACCGTGGTTGTGGTTATTTGAACAAGTTG GGCAGAGTCGTCGTCCCATCATTAACTACTCTGGTGGCACAGAAATTGGTGGTGGCATC CTGGCGTGTTTTCCCGGTCTGCCACAAAAGCCTTGTGCGTTTGACGGTCCAAT 


\begin{abstract}
AAaas CAAAAGCCTTGTGCGTTTGACGGTCCAATTCCGGGCATGGCCGTCGAGGTCCTGGATTC -G3 GGATGGTCGTCCTGTGCGCGGTAGCGTAGGGGAACTGGTCATTCGCCAGCCGTGGCCA GGTATGACCCATGGCTTCTGGCGTGACACCGAACGCTACCGTGAAGCATATTGGAGCGT CTGGCCAGATGTGTGGGTCCATGGAGATTGGGCACGTGTGGACGCAGATGGCTATTGG TTCGTGCATGGTCGCAGCGATGATACCATTAAAATTGCAGGGAAGCGCGTTGGTCCGGC AGACTTTGAAAGTGCTTTGGTCTCACATCCATTGGTGGTAGAGGCTGTTGCGGTGGGTG TACCAGATGAGTTAAAAGGCGAAACGGCAGTATGCTTTGTGACCGTTGCCGACAACGAA GCATTGGCAGCACGTCCGTGGCAAGCCTGGGAAGCTGAACTCGTGGATCACGTTGGTC GCCAGTTAGGTAAACCTTTACGTCCAGCTCATGTGCATCGCGTTGAAGCCATCCCTAAAA CCCGTAATGGTAAAACGGTACGTCGCGTAATGCGTAATGCCTATTTGGGGACCGCGCTG GGTGACCTTAGTTCGATTGAAAACCCAGCAGCTATCGATGCTGTGAGCGCTCTGCGTCC GTCTGCACAGGCAGTTCCCGACGCGATTGGCACTcatatgctcgaggatccggctgctaacaaa
\end{abstract}

\begin{tabular}{|c|c|}
\hline $\begin{array}{l}\text { RNaas } \\
-G 1\end{array}$ & $\begin{array}{l}\text { gaaggagatataccATGTCCAAACTCGCACGCTTGGAGCGTGAAGAGATTATGGAATGTCAAG } \\
\text { TGATGTGGGACCGGACTCTAAAAAAGACACCCAGATGGATCGTTTTCGTGCAGCAGTA } \\
\text { GGCACAGCCTGTGGGTGGCCCTGGGAACTATGACGATTTATACCATTGGAGCGTGCG } \\
\text { CTCATATAGCGATTTTGGGCAGATTCTGGAATTCTCGGGTATGTGTGCTCACGCAT } \\
\text { GTATGATGAGGTCGTGGATACCTCCAAGGGGTTGCTGACGTTCCTGAGTGGTTTCGTG } \\
\text { GGTCGCGTTTAACTACGCCGAAATCTGCTGCGCCACAAAGAAACGATCGCGTTGCC } \\
\text { CTCTACGTGGCACGTGAGGGTCGCGAAGAGATTCCAAAGTGACCTTCGAGGAACTGC } \\
\text { GTCAACAGGTTGCCCTCTTCGCAGCAGCTATGCGCAAAATGGGTGTCAAGAAAGGTGAT } \\
\text { CGCGTGGTCGGATACTTACCCAACTCTGCCCATGCGGTCGAAGCGATGCTGGCAGCAG } \\
\text { CAAGCATCGGTGCGATTTGGAGCTCAACCAGTCCGGATTTCGGCGTTAATGGCGTATTA } \\
\text { GACCGCTTCTCGCAGATTCAGCCGAAACTGATTTTCTCTGTTGAAGCAGTGGTCTACAAC } \\
\text { GGCAAAGAACATGGACACCTGGAGAAACTGCAGCGTGTGGTCAAAGGCCTCCCAGATC } \\
\text { TGCAACGCGTCGTATTGATC }\end{array}$ \\
\hline $\begin{array}{l}\text { RNaas } \\
-G 2\end{array}$ & $\begin{array}{l}\text { TCCCAGATCTGCAACGCGTCGTATTGATCCCGTACGTGTTGCCACGCGAAAAGATCGAC } \\
\text { ATCTCGAAGATTCCGAATAGCATGTTTCTGGATGATTTTCTGGCTTCTGGCACGGGTGCC } \\
\text { CAAGCACCTCAGCTGGATTTGAACAACTGCCCTTCAGTCATCCGCTCTTCATCATGTTT } \\
\text { TCTTCTGGTACCACCGGTGCACCGAATGCATGGTGCACTCTGCAGGCGGACACTGAT } \\
\text { CCAGCACCTGAAAGAGCACGTCCTGCATGGTAACATGACCAGCAGCGATATCTTACTTT } \\
\text { ACTATACCACTGTGGGCTGGATGATGGAACTGGATGGTCAGTGCTTAGCAACCGGT } \\
\text { GCCAGTTTAGTCTTATACGATGGGTCGCCTCTGGTGCGACTCCGACGTCCTCTGGGA } \\
\text { TTTAGTCGATCGCATTGGTATCACCATCTTAGGGACCGGTGCAAATGGCTGAGTGTCT } \\
\text { GGAAGAGAAGGACATGAAACCGATGGAAACCCACAACCTGCACACACTCCATACAATCC } \\
\text { TCTCAACTGGCTCTCCGTTAAAAGCCCAAAGCTATGAATATGTTTATCGCTGTATTAAATC } \\
\text { TACCGTGCTCCTGGGTTCTATTTCGGGTGGTACAGATATTATCTCATGCTTTATGGGTCA } \\
\text { AAACAGTAGCATTCCAGTCTACAAAGGAGAGATCCAAGCACGTAATC }\end{array}$ \\
\hline $\begin{array}{l}\text { RNaas } \\
\text {-G3 }\end{array}$ & $\begin{array}{l}\text { TCTACAAAGGAGAGATCCAAGCACGTAATCTGGGTATGGCAGTGGAAGCCTGGGATGAA } \\
\text { GAAGGCAAGACCGTATGGGGTGCATCTGGCGAACTCGTTTGCACCAAACCTATCCCGTG } \\
\text { TCAGCCGACTCATTTTTGGAACGATGAAATGGTCTAAATATCGTAAGGCGTACTTTAG } \\
\text { CAAGTATCCAGGCGTTTGGGCACATGGTGATTACTGTCGCATCAACCCGAAACGGGTG } \\
\text { GTATTGTGATGTTGGGTCGTTCGGACGGACCTTAAACCCAACGGTGTCGTTTGGC } \\
\text { TCCAGCGAAATCTATAACATCGTGGAAGTTTCACGAAGTCGAGGATTCATTGTGCGT } \\
\text { GCCACAATATAATCGTGATGGTGAAGAACGCGTTGTGCTGTTCTCAAAATGGCGTCCG } \\
\text { GTCATACCTTTCAGCCGGATTTGGTGAAACATATCCGCGATGCTATTCGTCTGGGTCTGT } \\
\text { CCGCTCGCCACGTTCCATCCTTAATTTTGGAGACACAGGGGATTCCGTATACGATCAATG } \\
\text { GCAAAAAAGTGGAGGTTGCAGTGAAGCAGGTAATCGCAGGCAAAACTGTTGAACATCGT } \\
\text { GGTGCTTTTTCCAACCCTGAGAGCCTGGATCTCTATCGCGATATTCCGGAATTACAAGAC } \\
\text { TTTcatatgctcgaggatccggctgctaacaaa }\end{array}$ \\
\hline
\end{tabular}




\begin{tabular}{|c|c|}
\hline $\begin{array}{l}\text { XAacx } \\
\text { A-G1 }\end{array}$ & $\begin{array}{l}\text { taccaggagatataccATGAACGTTCCGGTCGGACACCTGCGCAATGTCCAAGTTCTCGGAATC } \\
\text { GATGCAGGTGGTACGATGACCGACACGTTTTTTGTCGACCAAGATGGCGATTTTGTAGTT } \\
\text { GGGAAAGCTCAGTCCACTCCACAGAATGAAGCGCTTGGTCTCATTGCATCCTCCGAAGA } \\
\text { CGGTCTGGCCAACTGGGGAATGAGCCTGCACGAAGCGTTAGCCCAATTGCAGACCGGT } \\
\text { GTTTACTCTGGCACTGCGATGCTGAATCGCGTAGTTCAGCGCAAAGGTTTAAAATGCGG } \\
\text { TCTGATTGTGACCGTGGTATGGAGACTTCATCGCATGGGTCGTGGGTTCAGTCTC } \\
\text { ACTTAGGCTATGCTTATGAAGATCGCATCCATCTTAATACCCACCGCTACGACCCGCCGT } \\
\text { TAGTTCCGCGTCATTTGACGCGTGGTGTGTCGAGCGCACCGACATGATTGGTCCCAA } \\
\text { GTAATTCCACTTCGTGAAGACACCGCACGCGACGCAGCACGTGATCTCATTGCTGCTGA } \\
\text { TGCGGAAGGCATCGTAATCAGCTTGCTCCACTCGTATAAAAACCCTGAAAATGAACGCC } \\
\text { GTGTTCGCGATATTGTTCTGGAAGAGGTGGAAAAAATGGGAAAAAGATTCCGGTTTC } \\
\text { GCCTCTGCGGACTATTATCCTGTACGTAAAGAAACCCATCGTACCAACACGACCATTCTG } \\
\text { GAAGGTTATGCAGCGGAACCTTCT }\end{array}$ \\
\hline $\begin{array}{l}\text { XAacx } \\
\text { A-G2 }\end{array}$ & 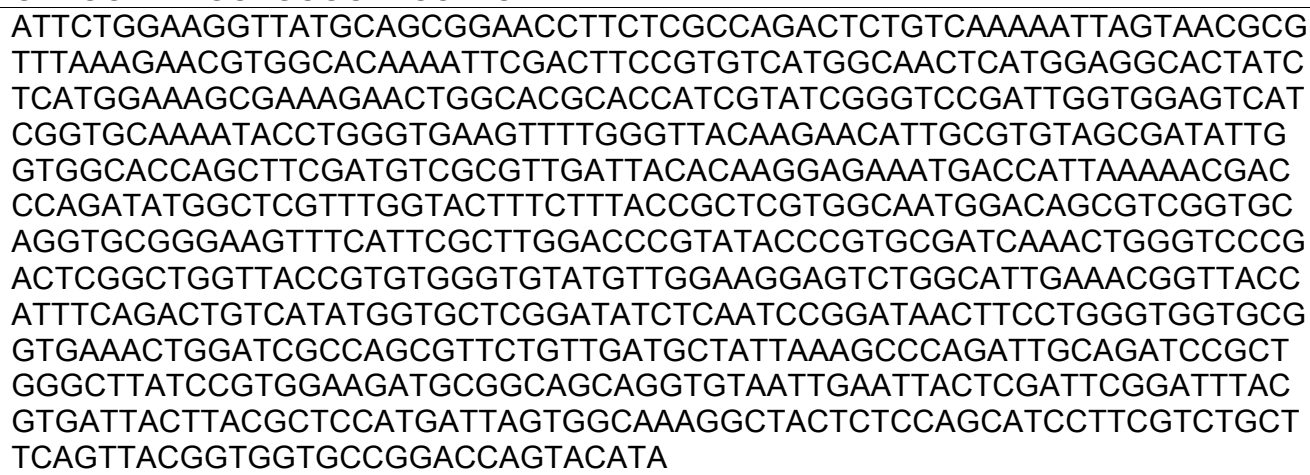 \\
\hline $\begin{array}{l}\text { XAacx } \\
\text { A-G3 }\end{array}$ & $\begin{array}{l}\text { TCAGTTACGGTGGTGCCGGACCAGTACATACCTATGGCTACACAGAAGGTCTGGGCTTT } \\
\text { GAAGACGTTATTGTGCCTGCGTGGGCTGCTGGCTTCAGTGCGTTCGGGTGCGCAGCTG } \\
\text { CAGACTTCGAATATCGTTATGATAAAAGCCTGGATATTAATATGCCGACCGAAACCCCTG } \\
\text { ACACCGATAAAGAAAAAGCTGCAGCAACGTTACAGGCAGCGTGGGAAGAGCTGACCAA } \\
\text { GAATGTCCTGGAAGAATTAAATTGAATGGCTACTCCGCAGACCAGGTTACCCTGCAACC } \\
\text { TGGTTATCGCATGCAGTATCGTGGTCAGCTGAATGATCTGGAAATTGAAAGCCACTGG } \\
\text { CCCAAGCCCACACCGCAGCCGACTGGGACAACTTACCGATGCGTCAATCTACTTAT } \\
\text { GGACGTGTGTACGCAGCAAGTGCGCGTCTCCAGAACTCGGATACAGCGTCACAGGTG } \\
\text { CGATTATGCGTGGCATGGTGCCTATCCCGAACCGAAAACCCAAAAGACCGGAAGAA } \\
\text { GGCGAAACACCTCCGGAGAGCGCTAAAATTGGTACCCGTAAATTTACCGTAAAAAACG } \\
\text { CTGGGTGGATGCCCAGTTGTACCATATGGAATCTTTACGTCCAGGCAACCGTGTTATGG } \\
\text { GTCCTGCTGTTATTGAAAGCGACGCGACGACATTTGTCGTACCGGACGGCTTTGAAACT } \\
\text { TGGTTGGATGGGCATCGCCTCTTTCATCTCCGTGAGGTTTAA }\end{array}$ \\
\hline
\end{tabular}

XAacx tctagaaggagatataccatgATGAATGTGACCGTTGACCAAAGTACCTTAGCAGGCGCTACTCGT B-G1 GGGATTGTTCGTGGTGGTGAAACCCTGAAAGAGCATCGTGACCGTCTGATGGCAGCAAC CAAGGCGACCGGACGCTATGCAGGGTTAAAAACCCTTGAACTTCGTGAACGCGAACCCA TCCTGTACAATAAGCTGTTTAGCCGTCTCCGTGCAGGTGTTGTTGACGCTCGCGAAACG GCTAAGAAGATTGCAGCGTCTCCGATTGTGGAACAGGAAGGGGAACTGTGTTTCACGCT TTATAACGCTGCTGGAGATTCCTTACTGACCTCCACGGGAATCATCATCCACGTGGGGA CGATGGGTGCAGCGATCAAGTACATGATCGAGAACAACTGGGAGGCTAATCCGGGTGT CCATGATAAAGATATCTTCTGTAACAATGATAGCCTTATCGGCAATGTCCACCCGTGCGA TATTCACACAATCGTACCGATTTTTTGGGAAGGTGAGTTGATCGGCTGGGTAGGTGGTG TGACCCATGTAATTGATACTGGTGCAGTTGGACCAGGTTCAATGGCTACCGGTCAGGTC CAACGCTTCGGTGACGGCTATTCCATTACCTGTCGTAAGGTAGGTGCGAATGATACGCT GTTCCGTGATTGGCTGCACGAAAGCCAGCGCATGGTACGCACCACCCGCTATTGGATGT TAGACGAGCGTACCC 


\begin{tabular}{|c|c|}
\hline $\begin{array}{l}\text { XAacx } \\
\text { B-G2 }\end{array}$ & $\begin{array}{l}\text { CGCTATTGGATGTTAGACGAGCGTACCCGCATCGCGGGGTGTCACATGATCCGCAAACT } \\
\text { GGTGGAAGAAGTGGTTGCGGAAGAGGGTATTGAGGCGTACTGGAAATTCGCGTATGAA } \\
\text { GCGGTGGACATGGTCGTTAGGGCTGCAAGCGCGTATTAAAGCAATGACCATCCCTGG } \\
\text { CACATACCGTCAAGTTGTTTCGTAGATGTTCCTTACGCTCATGAGGATGTCGCGTGCC } \\
\text { GTCGGACTTTGCGAACTGGATACGATTATGCATGCACCGTGCGAGATGACAATCCGCC } \\
\text { GTGATGGCACCTGGCGTCTCGACTTCGAAGGGTCCTCGCGTTGGGTTGGACACCTA } \\
\text { CAATGCCCATCAGGTATCCTTTACGTCTGGTATTTGGGTATGATGACGCAGACTCTGAT } \\
\text { TCCGAGCGAAATGATTAACGACGGTGCAGCCTATGGCACCGATTTCGTCTGCCAAAAG } \\
\text { GCACTTGGATGAATCCGGATGATCGTCGTGTGGCTTTTTCCTATTCCTGGCATTTCCTGG } \\
\text { TGAGTGCGTGGACCGCTCTGTGGCGTGGTCTTTCACGCTCCTATTTTGGTCGTGGGTAC } \\
\text { TTAGAGGAGGTTAATGCAGGGAACGCGAACACTAGCAATTGGCTGCAAGGTGGTGGCTT } \\
\text { CAACCAATACGACGAGATTCACGCAGTTAATAG }\end{array}$ \\
\hline $\begin{array}{l}\text { XAacx } \\
\text { B-G3 }\end{array}$ & $\begin{array}{l}\text { CCAATACGACGAGATTCACGCAGTTAATAGCTTTGAATGTGCTGCGAACGGCACTGGTG } \\
\text { CGACTGCCGTTCAGGATGGTTTGAGCCACGCTGCTGCTATTTGGAATCCGGAAGGAGAC } \\
\text { ATGGGTGATATGGAGATCTGGGAATTGCCGAACCGCTGGTTTATCTGGGTCGTCAGAT } \\
\text { TAAAGCAAGTTCTGGTGGAAGCGGTAAATATCGTGGAGGCTGTGGTTTTGAGTCACTCC } \\
\text { GCATGGTGTGGACGCGAAGATTGGACCATGTTTTTATGGGGACGGTCATATTTCAT } \\
\text { CGGATTGGGTCTGATGGTGGTACCCTGCAGCTTCTGGTTATCGCTTTGCTGCCCAC } \\
\text { AAGACTAATCTCAAGGAATTGATTGCGAGCGGTGCTGAATCCCGTTAGGTGGTATAC } \\
\text { CGATCCGGAAAACCGACCTGGGACGCCATGCTGCCAGATGCGCAGATCAAGCGTGAT } \\
\text { AAGCAAGCCATTACGACCGAGGAGATGTTCAGTGATTACGATCTCTATCTGAACTATATG } \\
\text { CGTGGTGGTCCAGGGTTTGGCGATCCGCTGGATCGTGAACCGCAAGCGGTTGCGGATG } \\
\text { ATATCAACGGTGGTTATGTCTTGGAACGCTT }\end{array}$ \\
\hline $\begin{array}{l}\text { XAacx } \\
\text { B-G4 }\end{array}$ & $\begin{array}{l}\text { TATCAACGGTGGTTATGTCTTGGAACGCTTCGCAGGTGAAGTCTATGGTGTTGTTGTGC } \\
\text { GTAAAGGTGCTGACGGTCAGTACGGTGTCGATGAAGCAGGTACTGCAGCAGCACGTGC } \\
\text { GCAAATTCGCAAAGATCGTTTAGCCAAAAGCGTTCCGGTTAGCGAATGGATGAAAGGTG } \\
\text { AGCGTGAAAAAATTCTCGCCAAGGATGCAGGGACCCAAGTTCGCCAGATGTTCGCTGCA } \\
\text { AGCTTCAAGCTCGGTCCTCGTTTTGAAAAGATTTTCGTACCTTTTGGAGCTTACCGGAT } \\
\text { AGCTGGACTCTGCCTGAAGAAGAATCGGTGTCCCACGTACGGTTCGCGCTATAGTAT } \\
\text { GGACATTTCCGAGTTACCGGACGTGCACACCGTGCAATTGTTGAAGAGTAAaggagatata } \\
\text { ccatggcg }\end{array}$ \\
\hline
\end{tabular}

XAacX ataaaggagatataccATGGCATATACCCGTTCGAAAATTGTTGACCTGGTTGACGGTAAAATC C-G1 GATCCGGATACCTTACATCAGATGTTATCTACACCGAAAGATCCGGAACGCTTTGTGACG TATGTGGAAATTCTGCAAGAACGCATGCCGTGGGATGATAAAATCATTCTTCCCCTGGGT CCGAAGCTGTTTATCGTTCAGCAGAAAGTTAGCAAAAAATGGACAGTACGCTGTGAATGT GGCCATGATTTTTGCGATTGGAAAGACAATTGGAAACTCTCTGCACGCGTACACGTGCG TGACACCCCACAAAAGATGGAGGAAATTTATCCGCGTCTGATGGCTCCAACTCCGAGCT GGCAGGTAATTCGTGAATACTTTTGTCCCGAGTGTGGCACGCTCCATGACGTCGAAGCA CCGACTCCGTGGTACCCAGTAATCCACGATTTTAGCCCTGACATTGAGGGCTTTTATCAG GAATGGCTTGGTCTGCCAGTTCCGGAACGTGCGGATGCGTAAgtcgacctgcaggcatgcaagctt

\begin{tabular}{|c|c|}
\hline Aacx & 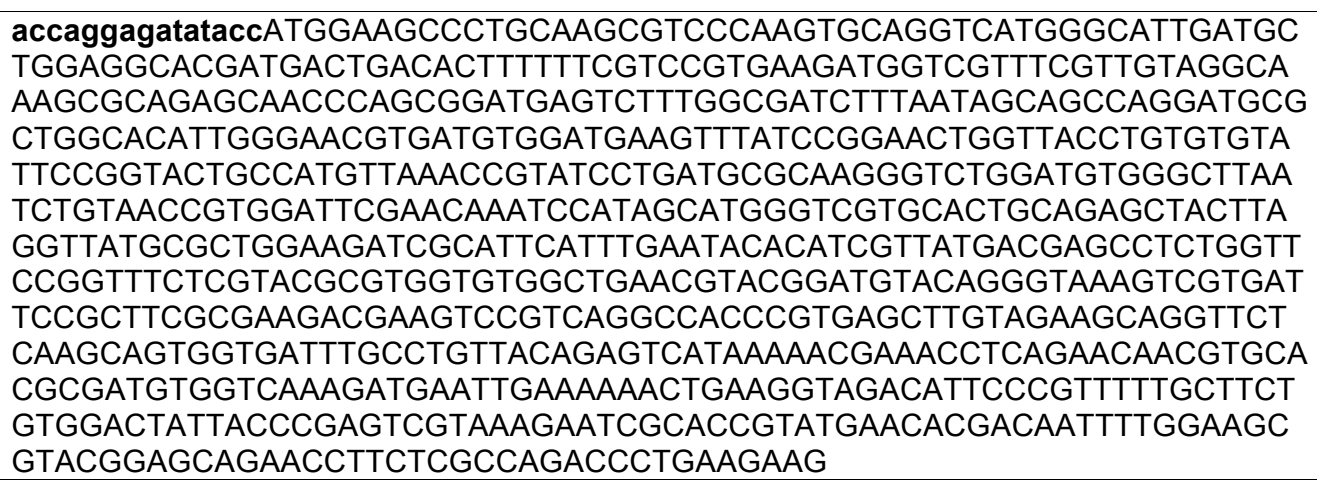 \\
\hline
\end{tabular}




\begin{tabular}{|c|c|}
\hline $\begin{array}{l}\text { AAacx } \\
\text { A-G2 }\end{array}$ & $\begin{array}{l}\text { CAGAACCTTCTCGCCAGACCCTGAAGAAGGTGAGCGATCGCTTCAAGAAACATGGAGCC } \\
\text { AAGTTTGATCTCCGTGTAATGGCTACGCACGGTGGTACGATCAGCTGGAAAGCCAAAGA } \\
\text { GCTTGCACGTACCATCGTGAGCGGTCCGATCGGTGGCGTGATCGGTAGTAAATTACTTG } \\
\text { GCGAATATCTGGGTGATGAGACATTGCGTGCAGCGATATGGAGGACCAGCTTCGAT } \\
\text { GTTGCTCTGATCACCAAAGGGTCGTTCGCGACAAAACGATCCCGATATGGCACGCCT } \\
\text { GGTACTGAGTCTGCCGTTGGTAGCAATGACTCTGTTGGCGCTGGTGAGGTTCGTTG } \\
\text { TGCGTCTGGATCCGTATAGTAAAAGCATTAAACTGGGACCGGATAGCGCAGGATATCGC } \\
\text { GTGGGCACGTGTTGGCCAGAATCTGGGCTGGACACCGTATCCGTGAGTGACTGCCACG } \\
\text { TGGTTCTGGGATACCTGAATCCGGATAATTTCTGGGTGGAGCGATCAAATTAGATGTAC } \\
\text { AGCGTGCACGCGACCATATTAAAGTCCAGATTGCCGATCCTCTGGGTCTTAGCGTCGAG } \\
\text { GATGCTGCGAGCGGTGTTATTGAACTGCTGGATCTGACGCTCTCGGAGTATCTCCGTGC } \\
\text { AAATATTTCAGCGAAAGGCTATAATCCAGCGGAATTTACCTGCTTCTCGTATGGTGGTGC } \\
\text { AGGACCGGTGCATACTTACGGCTATACCGAAG }\end{array}$ \\
\hline $\begin{array}{l}\text { AAacx } \\
\text { A-G3 }\end{array}$ & $\begin{array}{l}\text { ACCGGTGCATACTTACGGCTATACCGAAGGGGTGGGCTTCAAAGATGTCGTGGTGCCTG } \\
\text { CTTGGGCAGCGGGTTTCTCTGCGTTTGGCTGTGCATGTGCGGATTTCGAATATCGCTAC } \\
\text { GATAAATCCGTTGACCTGGGTGTCGCACAATTCGCGTCCGACGAAGTGAAAGCAGCAGC } \\
\text { GTGCGTCAGCCTGCAAGCAGCCTGGGAAGAATTAGCGGTAAAAGTAATTGACGAGTTCA } \\
\text { TTATCAACGGTTACAAACCTGAAGATGTATTGCTTATCCCAGGCTATAAGATGCAGTATAT } \\
\text { GGGCCAACTGAACGATCTGGAGATTGTATCTCCGGTGTCAACGGCAGCCACGGCAGGT } \\
\text { GACTGGAATCAGATTATCGAGGGTTCGAGACCACCTATAGTCGCGTTACGCTAATAG } \\
\text { CGCTCGTAGCCCTGAACTGGGCTTCTCTATTACTGGTGCTATCCTTCGTGGTATGGTTGT } \\
\text { GACCCAGAACCAGTTCTGCCTGAAGATGCTGACTGCGGTCCGACCCACCAAAGGAA } \\
\text { GCATATCTGGGAACCCGTCCGTCTATCGTCATAAACGCTGGGTCGAAGCCACTCTGTG } \\
\text { GAAAATGGAATCCTCAAAGCAGGTAACCACATTGTCGGTCCTGCGATTATCGAATCTGA } \\
\text { TGCAACCACCTTTGTGGTTCCGGATGGTTCGAGACTACGGTAGACAAGCATCGCCTGT } \\
\text { TTCACCTGAAAGAAGTGAAATAAtctagaaggagatatac }\end{array}$ \\
\hline
\end{tabular}

AAacx tctagaaggagatataccATGAATATGCTGACCAATAAAGAAGTTGGGTTCGCCGATCTGTTAA

B-G1 AAAATGGGCAGACACTGAAACAATTCCGTGATGGTATTATCGAACGTACCAAAGCCTCTG GCTGCTATAATGGTATCGAAAAGCTGGAATTTCGCGACTCGGATCCGATTGGCTATGAAA AACTGTTTTCGAAACTGCGTGGTGGTTTAGTGCACGCACGTGAGACTGCGAAGAAAATC GCAGCGTCTCCGATTGTGGAGCAGGAAGGGGAACTGTGTTTTACCTTGTACAACGCAGC AGGCGATTGCGTGCTGACCTCGACTGGCATCATTATCCATGTGGGTACCATGGGTGCAG CGATCAAATACATGATTGAGAACAATTGGGAAGGAAACCCTGGCATCAACCCACGTGAC ATGTTCACCACGAACGATTGTGCTATCGGCAATGTTCATCCGTGTGACATCGCTACCATC GTGCCGATTT

AAacx CGTGTGACATCGCTACCATCGTGCCGATTTTTTGGGAAGGCAAGTTAATTGGATGGGTG

B-G2 GGCGGTGTCACGCATGTGATCGACACGGGTGCAGTTACACCAGGCAGCATGTCGACGG GTCAGACCCAACGTTTTGGCGATGGCTATATGATTACGTGTCGTAAAACGGGTGTTAAC GATGAACCGCTGCGTGACTGGCTGCATGAATCCCAGCGTAGCGTTCGTACTCCGAAATA TTGGATCCTGGACGAGAAAACCCGTATTGCAGGATGCCATATGATTCGTGAATTAGTGG AAGAAGTTATCCGTGCGGATGGCCTTGATGCTTATGAGAAGTTTGGCTTTGAGGTTATTG AGGAAGGTCGTCGTGGATTGATGTCTCGTGTTAAAGCTATGACGCTTCCAGGAAAATATC GCAAAGTTAGCTTTGTTGACGTTCCCTTTAAGCATGAGGACGTACAAGTGAGCAATGCAT TCGCCAAACTGGACAGCATCA

AAacx CAATGCATTCGCCAAACTGGACAGCATCATGCACTCACCAGTTGAGATGACCATCAAAC B-G3 CCGATGGTAAGTGGCGTTTAGACTTTGAAGGTGCAAGCCGCTGGGGATGGCACACCTTC AACGCTCACCAGGTGGCCTTCACGAGCGGTATTTGGGTCATGATGTCCCAGACGCTGGT TCCGACTCAACGTATCAACGATGGAGCCTACTTTGCCACGGAATTTAAACTGCCAAAGG GTACATGGTGTAATCCTGACGATCGTCGCACTGGGCATGCGTACGCATGGCACTTTTTAA GTGTCAGGTTGGGCTGCCCTGTGGCGTGGCCTTAGTCAATCTTACTTCTCTCGTGGCTA TCTGGAGGAAGTGAACGCAGGTAATGCTAACACGTCGAATTGGCTTCAAGGTGGTGGCA TCAATCAGGATGGTGAGATTCATGCCGTAAACTCTTTTGAGGCGTCCTCGTGTGGAACG GGAGCCTGTGCGGTGAAAGATGGCCTTAATCACGCAGCAGCGATTTGGAATCCGGAAG GCGATATGGGTGACATCGAAATCTGGGAGATGGCTGAACCGCTCTTATATCTGGGTCGC AACGTTAAAGCGAACTCTGGAGGCTACGGGAAATATCGTGGTGGCTGTGGCTTTGAAAC CCTGCGCATGGTCTGGAATGCACAAGACTGGACCATGTTTTTCATGGGCAATGGTTATAT GAATAGTGATTG 


\begin{tabular}{|c|c|}
\hline $\begin{array}{l}\text { AAacx } \\
\text { B-G4 }\end{array}$ & 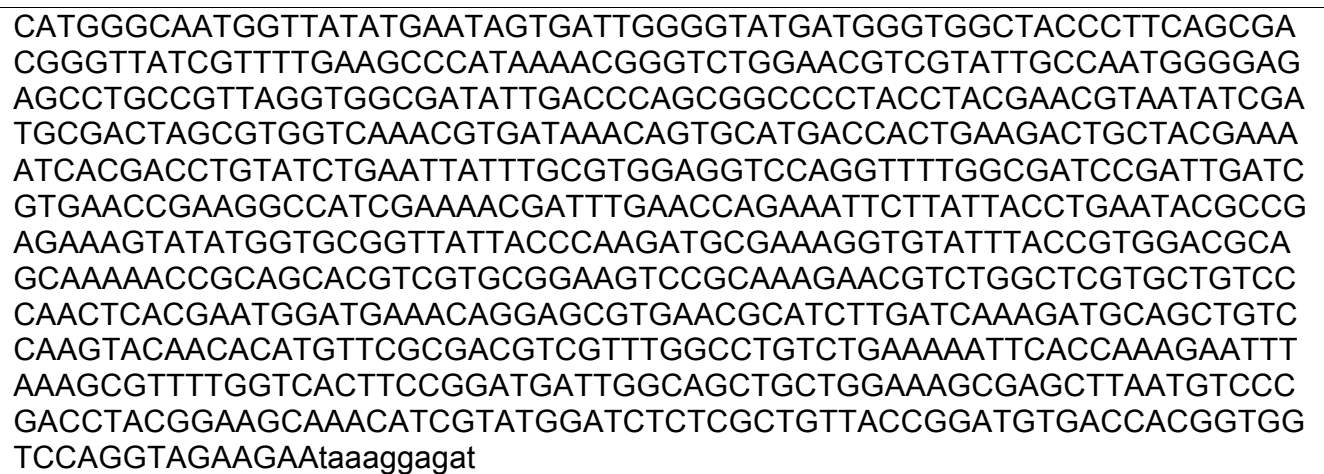 \\
\hline $\begin{array}{l}\text { AAacx } \\
\text { C-G1 }\end{array}$ & $\begin{array}{l}\text { tataccATGTCAACGTATACGAACGAACAGGTGGCTCATCTGGTTGAAGGTTCACTTGATT } \\
\text { GGGAAACGACTTTTCGCATGCTGTCAATGCCGAAAGACAATTCTCGCTTCGAACAGTATC } \\
\text { TGAAAGCCCTGCAAGCGAAATGAGCTTTCCAGACAAATTGTGCTCCCGTTGGGTCG } \\
\text { CACTTGTACATTGTCCAGTCGGCAAAACCAAACAATGGGTCACCATGTGTGACTGTGG } \\
\text { GCACGTGTTTGGGATTATCGCGAAACTGGAACTGAACGCAGCAATCTATGTTCGCG } \\
\text { ATACCGAGGAGGCAATGACCGAAGTTATCCGAAGCTGATGGCTCCGGATACCCAATGG } \\
\text { CAGGTTTATCGCGAATATTATTGCCCAACCTGCGGTACTATGCATGACGTGGAAGACC } \\
\text { GACCCCTTGGTATCCGGTTATTCATGATTTTGACCGGACATCGAAGCGTTTACCAGGA } \\
\text { ATGGGTAGGACTGCCTGTTCCCGAACGTGCAGCATAAgtcgacctgcaggcatgcaagettggctg } \\
\text { ttttg }\end{array}$ \\
\hline
\end{tabular}

\section{Literature Cited.}

(1) Lee, T. S., Krupa, R. A., Zhang, F., Hajimorad, M., Holtz, W. J., Prasad, N., Lee, S. K., and Keasling, J. D. (2011) BglBrick vectors and datasheets: A synthetic biology platform for gene expression. J. Biol. Eng. 5, 1-14. 